\title{
A Review on Image Reconstruction through MRI k-Space Data
}

\author{
Tanuj Kumar Jhamb \\ Master Degree Scholar, Department of Computer Science and Engineering, National Institute of Technology Calicut, \\ Kerala-673601, India \\ Email: tkjakaa@gmail.com \\ Vinith Rejathalal \\ Faculty, Department of Computer Science and Engineering, National Institute of Technology Calicut, Kerala-673601, \\ India \\ Email: vinithr.1981@gmail.com

\begin{abstract}
Dr. V.K. Govindan
Professor, Department of Computer Science and Engineering, National Institute of Technology Calicut, Kerala-673601, India

Email: vkg@ nitc.ac.in
\end{abstract}

\begin{abstract}
Image reconstruction is the process of generating an image of an object from the signals captured by the scanning machine. Medical imaging is an interdisciplinary field combining physics, biology, mathematics and computational sciences. This paper provides a complete overview of image reconstruction process in MRI (Magnetic Resonance Imaging). It reviews the computational aspect of medical image reconstruction. MRI is one of the commonly used medical imaging techniques. The data collected by MRI scanner for image reconstruction is called the k-space data. For reconstructing an image from k-space data, there are various algorithms such as Homodyne algorithm, Zero Filling method, Dictionary Learning, and Projections onto Convex Set method. All the characteristics of $\mathrm{k}$-space data and MRI data collection technique are reviewed in detail. The algorithms used for image reconstruction discussed in detail along with their pros and cons. Various modern magnetic resonance imaging techniques like functional MRI, diffusion MRI have also been introduced. The concepts of classical techniques like Expectation Maximization, Sensitive Encoding, Level Set Method, and the recent techniques such as Alternating Minimization, Signal Modeling, and Sphere Shaped Support Vector Machine are also reviewed. It is observed that most of these techniques enhance the gradient encoding and reduce the scanning time. Classical algorithms provide undesirable blurring effect when the degree of phase variation is high in partial k-space. Modern reconstructions algorithms such as Dictionary learning works well even with high phase variation as these are iterative procedures.
\end{abstract}

Index Terms-MRI, NMR, Modern MRI, k-Space, Fourier Transform, Phase Error, Phase Correction, SURE, SSSVM.

\section{INTRODUCTION}

There are scanners [1] which help to analyze the patient's whole body or part of it, after analyzing, they render high quality images which have detailed information about the scanned part. Some of the scanners are MRI, CT, PET (Positron Emission Tomography Doppler Ultrasound, and SPECT (Single Photon Emission Computed Tomography). They differ in the modality employed in capturing the images. Over the past two decades, the cost for these devices is reduced and easily affordable to hospitals and diagnostic centers.

MRI [2] is used to generate detailed and informative images of the tissues and organs within the patient body with the help of radio waves and magnetic field. It helps doctor to recognize the various medical characteristics of the scanned part of patient body in detail with the help Nuclear Magnetic Response (NMR) signals, discovered by Bloch and Purcell who shared the Nobel prize in physics for this discovery in 1952 [3]. MRI is a noninvasive [4] scanning technique. Non-invasive refers to the analysis of the diseases in human body without inserting any instrument inside the body. It does not use ionizing radiations [5] that are harmful to human body and hence it is preferred [6] over Computed Tomography (CT) scan for examining soft tissues. MRI produces higher resolution images of soft tissues compared to CT.

MRI makes use of NMR of hydrogen nuclei. After the generation of NMR signals, they are picked to form kspace data with the help of Radio Frequency Coil. MRI scanning machine includes hardware components [8] like Magnets, Gradient Coils, RF Coils, RF Detector, computer system, and safety tool box.

\section{A. Scope and Challenges}

The raw data obtained from scanning devices depends on the type of scanning device. K-space data [9] is 
obtained from MRI and Projection [10] data set is obtained from CT scan devices. The raw data (k-space data) may contain some missing entries because of improper scanning or due to traveling in medium (air or vacuum) or due to some other causes. The quality of raw data affects the quality of final reconstructed images in all the scanning devices. The major issue in MRI is the reconstruction of an image from the partial k-space (incomplete) data produced by the MRI scanner. It is very difficult to have a scanning device that can generate complete k-space data. The problem of missing or noisy $\mathrm{k}$-space data is overcome by devising computational procedures that can correct the data before applying reconstruction algorithms. Hence, the problem of reconstruction of an image from $\mathrm{k}$-space is a challenging one.

\section{B. Applications}

Magnetic Resonance Imaging and its variants like Diffusion Tensor Imaging, and Functional MRI are widely used in both research and diagnosis in Medical Imaging. Different modalities reveal different aspects of the scanned part. For instance, in neuro-imaging T1, T2 and Fair images [12] give high-resolution depiction of the structural features of the brain. They serve as very efficient diagnostic methods to identify the tumors [13] and other structural deformations [14] and enable perfect tracing of the contours of the abnormalities [15]. This is of great assistance in surgical removal [16] of them, as it requires exact demarcation of the boundaries.

Diffusion Tensor Imaging (DTI) captures the structural connections in the brain by imaging neural fibers. DTI mainly gives a clear understanding of white matter. DTI works by estimating the principal direction of water diffusion at each voxel position, by designing an ingenious sequence of application of the magnetic gradients. The reconstruction process is different from that used in normal MRI and is beyond the scope of the paper. The reader is advised to refer to [30] for detailed discussion on DTI.

In research area, MRI has been a revolutionary tool in neuroscience [17] research and has helped to unravel the complex neurological processes underlying many cognitive responses. It has lead to many leaps in brain connectivity analysis which studies the functional connectivity between different regions in the brain which may or may not be structurally wired together. It helps to understand the synergy that exists between distinct functional regions of the brain [19] in carrying out different psycho-somatic responses.

The rest of this paper is structured as follows: In Section II, the fundamental principles of MRI are presented. Section III discusses the modern MRI techniques and Section IV deals with the k-space data in detail. The classical techniques and modern techniques in image reconstruction along with algorithms are reviewed in from section V to section VIII. Finally, the paper is concluded in Section IX summarizing the outcome of the review.

\section{FundAMENTAL PRINCIPLE OF MRI}

MRI uses the principle of nuclear magnetic resonance. Spatial Information is provided by the spatial gradient of the applied magnetic field. The sampling trajectory in the $\mathrm{k}$-space is determined by the time dependence of the applied magnetic field gradient. The engineering aspect of MRI lies in properly designing the way the magnetic field is applied to evoke nuclear magnetic resonance to capture the spatial distribution of hydrogen protons in the body. Three aspects are discussed in the following sections.

\section{A. Magnetization Concept}

There are three kinds of magnetic fields [20] in the MRI scan machine: (i) $\mathrm{B}_{0}$-main magnetic field, (ii) $\mathrm{B}_{1^{-}}$secondary magnetic produced by Gradient coil, (iii) Radio frequency pulse.

Primary magnetic field aligns hydrogen atom in parallel of anti-parallel direction that is called longitudinal magnetization as shown in Fig. 1. Gradient coils produce secondary magnetic field; there are three gradient coils along $\mathrm{x}, \mathrm{y}$ and $\mathrm{z}$ axes. It allow MRI to produce magnetic field directionally along $\mathrm{x}, \mathrm{y}$, and $\mathrm{z}$ axes. It also allows spatial encoding for MRI images in the $\mathrm{x}, \mathrm{y}$ and $\mathrm{z}$ axes that is also called localization.

An object is located in the main magnetic field; net magnetization is developed by gradient magnetic field along $\mathrm{x}, \mathrm{y}, \mathrm{z}$ axes.

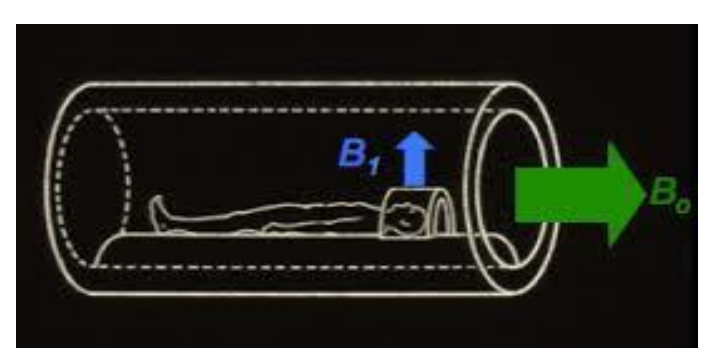

Fig.1. Magnetization in MRI Scan Machine [20, 60]

Net magnetic moment [20] can be given as:

$$
\mathrm{B}(\mathrm{r})=\mathrm{B}_{0}+\mathrm{G}_{\mathrm{r}} \cdot \mathrm{r}
$$

$\mathrm{RF}$ coils is used for transmitting RF pulse and receiving signals in MRI, it also disturbs alignment of protons. Some low energy protons spin to a high energy state by reducing magnetization in longitudinal direction.

A proton resuming to its normal state in the primary magnetic field is called Relaxation. Relaxation in longitudinal axis is termed as $\mathrm{T} 1$ relaxation (parallel to $\mathrm{B} 0$ ), and relaxation in transverse axis is termed as T2 relaxation (perpendicular to B0). After applying the RF pulse, the several protons get flipped back to low energy state from high energy state that is parallel to $\mathrm{B}_{0}$. This release in energy is obtained in the form of NMR signals. $\mathrm{RF}$ coils receive these signals.

The computer system accepts the analog data from RF coils and performs analog to digital conversion of data. The digitized version of the body part which will get 
stored in temporary image space also called the k-space, the Fourier transform of the image. K-space stored digitized NMR signals during data collection/ acquisition phase. It represents the spatial position covered by frequency and its phase data generated by MRI scan. KSpace data [21] is sent to image processor where some reconstruction algorithm is applied to reconstruct an image.

\section{B. Theory of Nuclear Spin}

The physical basis of NMR signals is the concept of Nuclear Spin [22]. NMR signals are generated when the protons come back to low energy from higher energy. Nuclear spin is an inherent form of angular momentum $(\mu)$ carried by atomic nuclei and other elementary particles [22]. Here, in MRI, It is an assumption that Hydrogen Atom nuclei protons get aligned in anti parallel or parallel towards the main magnetic field as shown in Fig. 2.

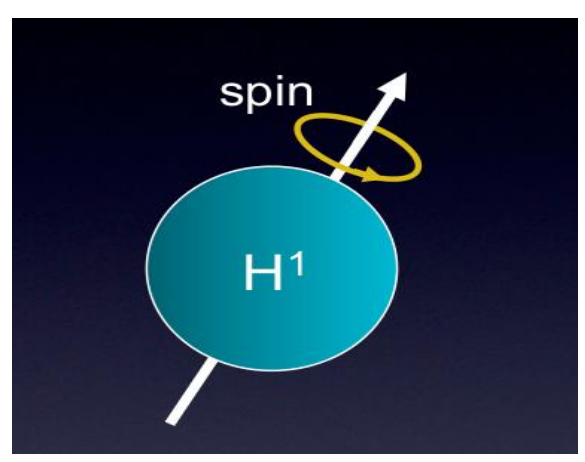

Fig.2. Hydrogen Atom Spinning [11]

The angular momentum [22] can be given as:

$$
\mu=\gamma \cdot s
$$

Where ' $\gamma$ ' is the magnetization "Gyromagnetic Ratio" and equation is called "Resonance Equation" [8].

\section{Microscopic Behaviour of Tissue Particles}

The human Body is composed [23, 24] of primarily with water and carbohydrates. The water and carbohydrates has bulk of hydrogen atoms. In normal human body, quantity of water is more than $60 \%$. When the gradient magnetic field fall on the body, the hydrogen atom of the body get excited to spin in one direction.

This spin [25] happens because Hydrogen Atom has only one proton and it could be aligned easily in MRI magnet. The proton possesses the property of spin which causes nucleus to generate an NMR signals that further catches by the radio frequency coils to generate k-space. Since, bones are not having any water; it does not generate any data for image. Bones leave blank area in images that is why MRI scanners are best for scanning soft tissues.

The Radio Frequency coils detect [26] the NMR signals generated by the nuclear spin. Both phase and frequency data are produced in the form of matrix, of complex numbers, whose central region has low frequency. This is generally referred as k-space data.

\section{MODERN MRI TECHNIQUES}

Modern MRI provides superior spatial and structural resolution of infections or diseases in human body. It gives the clear picture of temporal resolution of dynamic imaging and plays an important role in metabolic imaging. Here, a few of the MRI techniques are discussed as follows:

\section{A. Functional MRI}

The Functional MRI [27] was developed towards the end of 20th century. It is an invention that leads to many path-breaking researches in the field of cognitive sciences. It added a new dimension to the research by enabling the visualization of functional variations underlying the neural processes that manifest as the cognitive capabilities of individuals. fMRI has also served as a vital diagnostic tool by helping the identification of functional aberrations that impair the normal functioning of the brain. The technical aspects and applications of fMRI are briefly discussed below:

Neurological processes are basically electrochemical signals, which are the true indicators of brain activity which is hard to measure. Therefore, measurable surrogate indicators had to be developed to track neural activities and their derivations from equilibrium state.

In fMRI, typically a 3 Tesla magnet is used. fMRI measures the local change in blood flow by exploiting the fact that hemoglobin is diamagnetic when oxygenated and paramagnetic when deoxygenated. An increase in the volume of blood flow causes a small variation in the MR signal owing to a change in the concentration of oxygenated hemoglobin. This variation in MRI signal can be attributed to change in the level of neural activity.

Functional MRI is used to explain the functional movement of body part with respect to the brain. They are used to generate images of brain activity with the help of magnetization property. It helps to study the anatomy of human brain. It captures the magnetic differences in blood when it is in deoxygenated and oxygenated state [28]. In general, hemoglobin in the blood becomes paramagnetic when it is in de-oxygenated state, it produces larger Magnetic Resonance signal than in oxygenated state. It covers multiple images at various instances. While scanning, it enhances nerves to work faster and follow BOLD (Blood Oxygen Level Dependent) [29] effect, which is used for observing organs that are active (working) like brain tissues. Suppose if some portion of brain need to be removed because of tumor or any other cause, fMRI helps to analyze the side effects after removing that area from the brain.

\section{B. Diffusion MRI}

Diffusion MRI [30] is one of the growing techniques in MRI field. It estimates the scattering of water molecules in the tissue. This approach depends on the Brownian motion of water molecules. It provides structural information of muscles of Glands (e.g. prostate, thyroid etc.). Along with Diffusion MRI, DTI [31] technique is used for reconstructing images. Diffusion MRI [30] was 
developed for capturing images that are continuously varying, e.g., brain motion, cardiac condition etc. Diffusion MRI provides new insights in neurological research by shedding light on neural connections. It is used for scanning sensitive part of the body like heart muscles, nerves.

\section{Principles of Diffusion MRI (DMRI)}

Diffusion tensor imaging [32] is a relatively new development in the field of neuron-imaging; it is a variant of MR imaging. DTI utilizes the local diffusion of water, as shown in Fig. 3, in the brain to gather insights into the structural details of the brain. DTI banks on the tendency of water to diffuse more along the neural fibers or axons. In diffusion MRI, when the magnetic field gets excited, a dephasing gradient magnetic field is applied. This causes the magnetic dipoles at various locations to precess at different angular velocities. Dephasing magnetic field disrupts phase alignment and the signal captured is weakened consequently. If there is no motion of water molecules the application of a rephasing gradient should realign the magnetic dipoles. This realignment manifests as the strengthening of the signal captured. But, since the water diffuses during the rephasing process, the pulse fails to refocus all the magnetic dipoles resulting in a signal loss. The signal loss is a function of the diffusion properties of the tissue.

The principal direction of diffusion is obtained from the Eigen vectors of the diffusion tensor observed at that point. Water tends to diffuse more along the axon fibers, and hence, DTI can be used to infer the structural details of neural wiring.

\section{Real Time MRI}

Real Time MRI [33] scanning machine continuously monitors the objects in real time. Its imaging is based on radial iterative reconstruction and FLASH (Fast Low Angle Shot). FLASH [34] is a basic principle for measuring the $\mathrm{k}$-space data that is obtained from scanning device. It is applied to study cardiac movement of the nerves and obtain cardiovascular imaging. It allows obtaining the heart beat imaging that is up to 50 frames per second. Apart from cardiac activity it is also used for studying the joint kinetics.

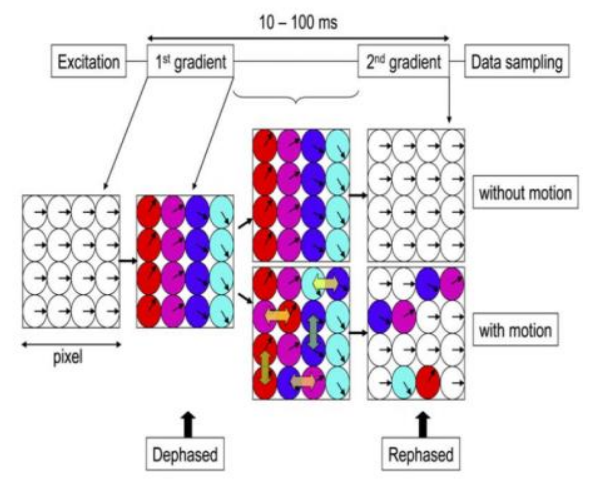

Fig.3. Illustrating dephasing and rephrasing in DMRI [30]

\section{Multinuclear Imaging}

Multinuclear Imaging [35] analyses the biological tissues which are having tremendous amount of Hydrogen. However, this Imaging technique is still not available for practical use outside research Labs; it is not available at diagnostic center or hospitals. It is used for determining nano-level distances among the nerves. It explains how nerves work.

\section{E. Interventional MRI}

Interventional MRI [36] was basically developed for the interventional radiology. It does not cause any harm to the patient while scanning. It has no magnets, but, it has quasi static fields and strong magnetic radio frequency fields that are generated from the scanner. Its degree of invasiveness (Section 1, Introduction) is quite high as compared to other MRI imaging techniques.

\section{F. $\quad$ Susceptibility Weighted Imaging (SWI)}

SWI [37] is different from the concept of spin density in MRI. It uses echo constraints, pulse sequences for capturing images. It utilizes the concept of magnetic susceptibility [38] for detecting the differences among various tissues. Each type of tissue has different echo gradient for magnetic field. It is applicable for detecting tumors, traumatic brain injury, stroke etc.

\section{G. Magnetic Resonance Spectroscopy (MRS)}

MRS [39] is used to measure the metabolism in the body tissues. Metabolism [40] is the reactions that allow living beings to grow and reproduce. MRS generates resonance spectrum that is related to the distribution of isotope that will excite to form raw data of the scanned object. Two or more elements are called isotope [41] when they have same number of protons in their nuclei. It is used for diagnosing metabolic disorder. It is also used for estimating biochemical reaction information of the tissue in the human body.

\section{K-SPACE DATA}

$\mathrm{K}$-space [42] data is related to image data by Fourier Transform. There are three independent working coils that provide gradient magnetic field along $\mathrm{X}, \mathrm{Y}$ and $\mathrm{Z}$ directions. More compactly we can say, gradient can be defined as vector in 3 dimensions.

The Larmor frequency $(\omega)$ [43] represents the angular momentum of precession generated by magnetic moment of the protons and can be given as:

$$
\omega=\gamma B
$$

$\mathrm{B}$ is the strength of the static magnetic field, and $\gamma$ is the gyromagnetic ratio.

In MRI, due to the presence of main magnetic field $\left(\mathrm{B}_{0}\right)$ and gradient magnetic field $(\mathrm{G})$, Larmor frequency (also called spatial frequency) can be calculated as: 


$$
\omega=\gamma\left(\overrightarrow{B_{0}}+\vec{G} \cdot \vec{r}\right)
$$

Where $\bar{r}$ is the spatial location (coordinates of a point) in the image. In rotating frame, the main magnetic field vanishes, $\omega$ can be given as:

$$
\omega(r, t)=\gamma(\vec{G}(t) \cdot \vec{r})
$$

Phase, $\phi$ is the integral of angular frequency. Phase of the image at $\mathrm{t}=0$ can be given as $\phi(0)=0$; at any time $\mathrm{t}$, it is given by

$$
\phi(r, t)=\int_{0}^{t} \omega(r, \tau) d \tau
$$

Substituting equation (5) in (6), it can be written as

$$
\begin{gathered}
\phi(r, t)=\gamma \int_{0}^{t} \vec{G}(\tau) \cdot \overrightarrow{\mathrm{r}} d \tau \\
k(t)=\gamma \int_{0}^{t} \vec{G}(\tau) d \tau
\end{gathered}
$$

Now $\phi(r, t)$ can be written as

$$
\phi(r, t)=k(t) \cdot r
$$

MRI scanner output can be represented as a function of time as

$$
s(t)=\int M_{x y}(r, t) d r
$$

$M_{x y}$ is the vector sum of all transverse magnetization exists in the image at any point of time. In MRI, the signal obtained as a function of $r$ and phase in the image. Phase varies with respect to both space and time:

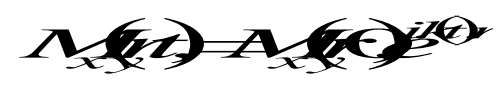

$\mathrm{M}_{\mathrm{xy}}(\mathrm{r}, 0)$ represents an Image at time; $\mathrm{t}=0$ as a function of spatial position, and $\mathrm{e}^{\mathrm{i} . \mathrm{k}(\mathrm{t}) \cdot \mathrm{r}}$ represents phase. Image cannot be obtained directly. Instead, it is obtained along with phase which changes over the region during scanning.

$M_{x y}(r, t)$ represents the image $f(r, t)$.

Substitute equation (11) in equation (10),

$$
s(t)=\int f(r, t) \cdot e^{i \cdot k(t) \cdot r} d r
$$

This equation is exactly the definition of Fourier Transform which tells that $s(t)$ provides the Fourier coefficients of the image $f(r, t)$.

\section{A. $\quad k$-Space Data Generation}

In MRI, reconstruction of an Image is more complex than other scanning devices like CT-scan and Ultrasound scan. Most of the k-space data reside in the lower frequency region (Fig. 4). The higher frequency regions are generally sparse in k-space, and most of the information of an image is present near the central region of k-space.

The Fourier Transform of a real signal is a conjugate symmetry [44]. Due to this reason the k-space data also follows conjugate symmetry. The high spatial frequencies correspond to the region of an image where the rate of change in intensity is more. The low spatial frequency corresponds to the region of an image where the rate of change in intensity is less.

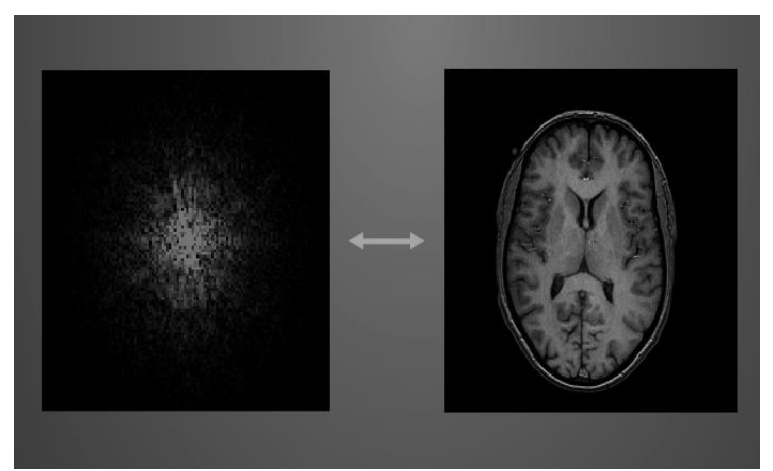

Fig.4. Inverse Fourier Transform of an k-space [65]

Reconstruction of an image from its $\mathrm{k}$-space data is one of the applications of Inverse Problem in Image or Signal Processing field.

The central region in $\mathrm{k}$-space provides information regarding the contrast [45] of the image, and the peripheral regions provide the detail about the structure like borders and contour of an image.

\section{B. $\quad k$-space Data and Partial k-space}

$\mathrm{K}$-space data can be referred as Full k-space data when there are no missing entries present in k-space or k-space data is completely available [46]. It is practically difficult to obtain the full k-pace because of non-uniform nature of the radio frequency field, primary field and gradient field. Ideally, the Fourier Transform of an image is equivalent to the full k-space data [46].

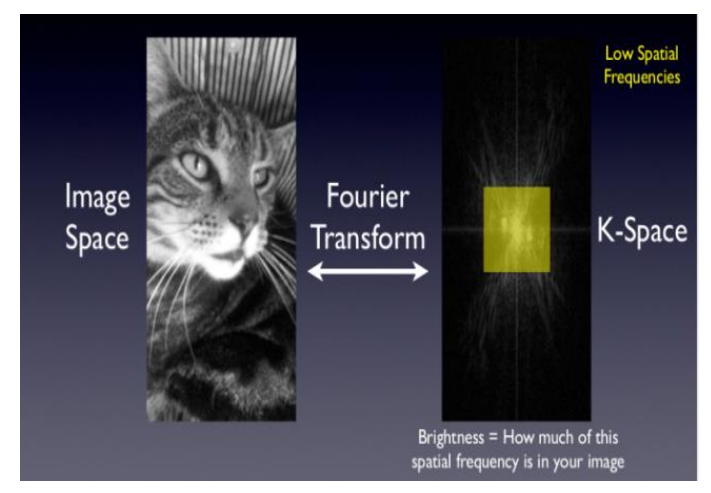

Fig.5. Fourier transform of an image gives full k-space data [47] 
By doing inverse Fourier Transformation, it is possible to get proper reconstructed image (Fig. 5). Though, the kspace data is not fully available, there exists symmetry in $\mathrm{k}$-space matrix; this symmetric nature of k-space enables rebuilding the missing $\mathrm{k}$-space data to a greater extent.

\section{Fourier Transform and k-Space Data}

More technically, k-Space data contain the density [47] of nuclear magnetic resonance signals generated from the body and it gets collected on the MRI radio frequency coil when the magnetic field get passed from the object.

The important information of an image is near to central region only. Magnetic Resonance Imaging analyzes [48] the Fourier transform of the spatial frequency entries in $\mathrm{k}$-Space data of hydrogen atom nuclei. Due to this phenomenon the $\mathrm{k}$-Space data is also a definition of Fourier transform. Example k-space data and the corresponding image are shown in Fig. 4.

\section{D. $k$-Space Imaging Sequence}

The Imaging sequence consists of four phases: data collection phase, preprocessing phase, reconstruction phase and post processing phase.

The process of collecting the partial k-space data from RF coil in MRI comes under the data collection phase. The collected k-space is analog in nature which is not amenable for processing by digital computers. Hence, reconstruction from $\mathrm{k}$-space is preceded by a preprocessing phase which converts the analog data to digital form. Conversion of the preprocessed k-space data into a visually interpretable and meaningful image is done in reconstruction phase. In the post processing phase, it is needed for removing noise in the reconstructed image with the use of filter concept, de-convolution, or total variance etc.

\section{E. MR and CT Imaging Data}

Computed Tomography (CT) collects the data in the form of projections while in magnetic resonance imaging it collects the data in the form of $\mathrm{k}$-Space. These projections are simulation of Radon transform [49] of an image in computed tomography while in magnetic resonance imaging; k-Space is the simulation of Fourier transform.

Both magnetic resonance imaging and computed tomography follow the concept of inverse problem via PST (Projection Slice Theorem) [50] in CT scan and PSF (Point Spread Function) [51] in MRI scan. In MRI, the data collected is not the projections but it is the spatial frequency information of imaged object. The conceptual difference between the modalities [52], which imply the method of reconstruction, is entirely different.

\section{F. Phase Correction}

There is always variation in phase while collecting kSpace data because of non-uniform distribution of magnetic field. Acquisition of the complete k-space is not feasible. The paper [51] divides the k-space data in several sub sections or plane and there exist one plane corresponding to each sub section. So, error varies when processing occurs from sub section to sub section that should be eliminated for constructing proper image. These sections are also called Blades [51]. These Blades make hermitian symmetric matrix. Each Blade is transferred into Fourier Space with the help of Fourier Transformation.

\section{G. Gibbs Ringing Artifact}

Gibbb's artifacts [51] are unwanted signals which usually appear near the edges in an image. Gibb's artifact gives ghosting in an image. It usually occurs because of the low pass filter. Here in k-space reconstruction it occurs due to the variation in phase.

When the k-space data is generated by Fourier Transform then most of the Reconstructed Image is affected by Gibbs Ringing Artifact [51]. During Reconstruction of an Image it accepts the some spurious signals (also say, noise) when the image reconstruction is in processing.

It occurs when we are processing/sampling only Low Spatial Frequency region of k-Space. This Artifact gives noise near the edge in an image because there is a transformation happen when it shifts from low spatial frequency to high spatial frequency or vice versa. So, there is some noise add while processing these areas. These artifacts can be prevented by using the concept of convolution as a function of smoothing.

\section{RELATED ClASSICAL WORKS IN MRI IMAGE RECONSTRUCTION}

Enormous amount of work has been done in the field of reconstruction of images from the signals acquired by the scamming devices. There has been staggering improvement in the quality of reconstruction since 1970 [53]. A few of the most notable works are reviewed in the following subsections.

\section{A. Expectation Maximization}

One of the classical works in image reconstruction is that by Cheng and Dewey [54]. Authors described the concept of reconstruction of image from projections or raw data as an inverse problem, and implemented for cases where large number of images to be reconstructed. They derived an algorithm that is much efficient than filtered layer-gram method for CT scans. By using highpass filter they formed a system of linear equations that can be evaluated very well in the Fourier domain. Expectation maximization is employed for determining the class values that are not available. MRF (Markov Random Field) model of dynamic objects is used to achieve local regularity and increase smoothness during reconstruction.

\section{B. Sensitivity Encoding}

Pruessmann et al. [55] proposed a new approach for collecting the k-space data refereed as SENSE (Sensitivity Encoding). In classical technique, Fourier Imaging collects the data on RF coil at very slow rate but 
with the help of SENSE we can enhance the capability of Fourier Imaging at the double rate. This also reduced the time for scanning. The normal encoding (that is, by Fourier Imaging) samples the k-space data and transfers it to the RF coil. It does not enhance the sampling density. If the Fourier Imaging is used with SENSE, it reduces the sampling density and collects the k-space more efficiently. SENSE enhanced the gradient encoding and reduced the scan time as compared to Fourier imaging.

\section{OTSUS Imaging Technique}

Borse et al. [57] presented the concept of threshold technique for imaging called OTSUS. It maximizes the intensity of pixels in order to perform picture thresholding on the clustering basis. This method is not good for segmentation. It is suitable for image reconstruction. This method is used to reconstruct 3D images from $2 \mathrm{D}$ images. If the raw data has too many real values, it is not possible for the software to estimate the expected value and will not work properly for these kinds of images. It gives a user friendly interface with good visualization. It was used by Doctors and Radiologists and allowed them to reconstruct large number of images from various angular visions.

\section{Inverse Problem and Modulation Transfer Function}

Andrei et al. [58] presented a new approach to reconstruct an image using inversion of exponential divergent transformation of beam analytically that could serve a mathematical model for 3D spectacular of cone beam imaging. The approach uses a circular scan on cone beam based on an assumption that an area which is convex can be attenuated constantly. The inverse problem is reduced to a restoration problem analytically with the help of PSF (point spread function) [59]. The method requires two processing steps for image reconstruction, namely, filtering projection and back projection. The Wavelet transform used provides good result for filtering the noise.

\section{E. Linear Inverse Problem}

Ribés and Schmitt [61] applied idea of inverse problem in image processing for reconstructing images. As we know, the inverse problem is a classical problem in Physics. Due to the continuous distribution of raw-data (k-space in MRI), they implemented concept of Linear Inverse Problem in image processing. Four techniques are proposed for the solution of linear inverse problem directly for the reconstruction of an image. The techniques are: Interpolation, Indirect Reconstruction, Direct Reconstruction and Fourier Transformation based Reconstruction. They also discussed the application of Inverse Problem in Magnetic Resonance Imaging and Multispectral Imaging [62].

Gylson and Govindan [56] discussed a fast algorithm for reconstruction computing CT (Computed Tomography) images. Filtered back-projection algorithm is implemented in various domains. The cost of computation and the RMS error of reconstruction are discussed. In [63], Gylson and Govindan also presented an efficient back projection method using Walsh Transform. The Walsh Transform method provides faster computation when compared to DFT.

\section{F. Level Set Method}

Chul et al. [64] discussed transform of discretized domain using gradient method of unique switching behavior which is used to stabilize evolution of level sets and the trade-off of constructing the signal/ image are hiked up with respect to pixel and their support. They also mentioned level set method [65] that increases the stability and quality of raw data. Level Set Method is a general concept of solving problems in Image Science, 3D graph theory applications etc. It numerically analyzes the various shapes and surfaces on fixed n-dimension coordinates. It also helps to analyze time varying object in real time. It works in per-processing phase of an image. It divides given continuous function into level sets and then analyzes each one numerically and returns the result to reconstruction algorithm.

\section{G. Compressed Sensing or Compressive Sampling}

Montefusco and Lazzaro [66] discussed primitive approaches for sampling of the signals/images that follow the fundamental of the NST (Nyquist sampling theory). This theory proofs that the rate of sample must be at least equal to twice of the utmost frequency present in the signal. This principle covers all the signals/images that are used to reconstruct final result from the projections like medical images and radio astronomy images. They discussed the basic two principles of Compressive Sampling. First, the signal must be compressible or sparse and have a precise representation when it is indicated in the form of basis; second, the collection of data should be non coherent with the sparsed basis and have dense description in that basis. Complete sampling allows reconstructing large number of images efficiently.

Lustig et al. [67] also presented the concept of Compressed Sensing in the context of reconstruction of images and signals from partial k-space data generated by MRI scanner. Using compressed sensing in MRI, it does data collection in two ways: (i), by Wavelet Transform [68], and (ii) by encoding spatial frequency constraint in the form of projection. They focused their discussion in the second way of data collection, that is, spatial frequency [69]. By collecting data using the concept of compressed sensing, they were able to collect the data in faster ways.

\section{H. Cone Beam Computed Tomography}

Kan et al. [70] discussed about the Image guided radiation therapy. This kind of therapy is very much required to ensure the correctness of the aspects using the discretized projections of tissue. They also discussed Cone Beam Computed Tomography (CBCT) [71] which is very popular online imaging tools for modeling tissue from CT scan or MRI scan. Although CBCT is much suitable to use, the presentation of CBCT systems is quite less than the default CBCT. The image quality for the $\mathrm{CBCT}$ can be dissipated very easily due to the change in 
medium while reconstructing from projections. The CBCT uses artifacts reconstructions, that is, approximate analysis of the image. In order to further get better the CBCT image quality and trim down the reconstruction artifacts, they used various iterative reconstruction algebraic approaches used for different CBCT geometries in their paper. They evaluated the artifacts of these algorithms using a variety of numerical methods as well as digitally projected images of long suffered people.

In the discussions carried out in this Section, many approaches for reconstructing images have been reviewed like Sensitivity Encoding, Level Set Method and many others. The basic idea of these approaches is being used as foundation for many recent research works also. Compressed Sensing is used for reducing the scan time even for current MRI scan machine. CBCT is also a great way of reconstructing images online. This is widely used by Bio Medical Universities for analyzing diseases through MRI scanned images online. Some of the most popular classical algorithms [72] from k-space data like Zero Filling Method, Phase Correction using Conjugate Symmetry, and Homodyne Reconstruction Algorithm for reconstruction are discussed in detail in the next section (VI). All these classical algorithms are direct methods requiring only one pass for the processing.

\section{Classical Algorithms For IMAGE RECONSTRUCTION}

Image Reconstruction is the process of converting $\mathrm{k}$ space data to an image. The partial $\mathrm{k}$-space data is obtained from MRI scan, this k-space is having some missing entries due to various reasons, as we already discussed. There exist many classical algorithms to reconstruct an image from k-space data like Zero Padding (or Filling) Method, Phase Correction using Conjugate Symmetry, Homodyne Algorithm. These all are the direct method (only one pass required for the processing) for image reconstruction.

\section{A. Zero Filling Reconstruction Algorithm}

As the name suggests zero filling [73], zero get filled in the missing entries of $\mathrm{k}$-space data. It is naive approach of reconstructing images. It is directly making missing entries to zero without rebuilding. It works well when almost full k-space data is available. The algorithm takes input as partial $\mathrm{k}$-space data and produces reconstructed image.

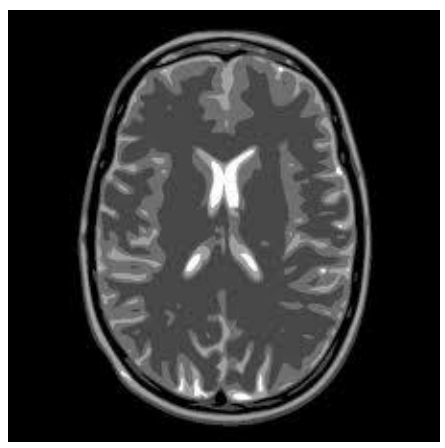

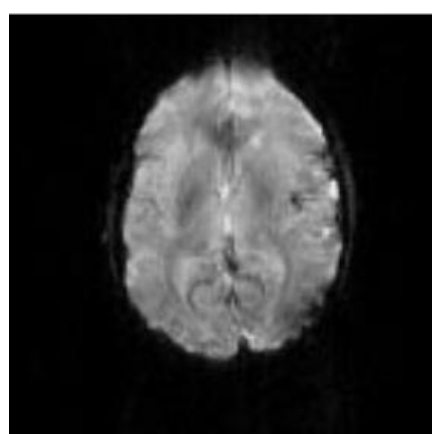

Fig.6. Original Phantom Image (U) and Reconstructed Phantom Image (D) after applying Zero Filling Algorithm

Pros: The best thing of this algorithm is that it is simple. If the full $\mathrm{k}$-space data is available, it gives reasonably good results.

Cons: It is practically difficult to obtain full k-space data, hence it cannot be used for reconstructing medical images and it is not an acceptable technique.

Result: The output image is blurred as we can see in Fig. 6, and it is not acceptable for the diagnostic purpose. It is needed to fill missing entries with some meaningful data.

\section{B. Phase Correction using Conjugate Symmetry}

It has been discussed in section (IV, F) about the phase correction, due to the phase error in k-space data set, it obtains a few missing entries. These entries required to fill for the proper image reconstruction. This method fills these entries by considering the property of conjugate symmetry of k-space. The algorithm takes input as partial k-space data and produces reconstructed image.

\section{Denotations:}

Symmetric data (Central Region): $\mathrm{M}_{\mathrm{s}}\left(\mathrm{k}_{\mathrm{x}}, \mathrm{k}_{\mathrm{y}}\right)$

Symmetric Data Reconstructed image: $\mathrm{m}_{\mathrm{s}}(\mathrm{x}, \mathrm{y})$

Partial k-space image obtained after IFT: $\mathrm{m}_{\mathrm{pk}}(\mathrm{x}, \mathrm{y})$

Algorithm [74]:

1) The phase estimate can be given as $p^{*}(x, y)=e^{(-i}$ $\left.{ }^{*} \phi(x, y)\right)$; and $\phi=\arg \left(m_{s}(x, y)\right) ; w$ here $\mathrm{p}^{*}(\mathrm{x}, \mathrm{y})$ is multiplied with image $\mathrm{m}_{\mathrm{pk}}(\mathrm{x}, \mathrm{y})$ and it gives phase corrected image. Apply Fourier transformation to this phase corrected data. It obtains phase corrected partial k-space data. In this algorithm, $\mathrm{m}_{\mathrm{s}}(\mathrm{x}, \mathrm{y})$ is used for the phase correction.

2) Apply Conjugate Symmetry on the phase corrected to get symmetric k-space data.

3) Apply Inverse Fourier transformation on the symmetric data set to get required Image.

Fig. 9 illustrates the algorithm

Pros: Images obtained from this algorithm are phase corrected image and the results are better than zero filling method.

Cons: It does not work when most of the k-space data entries are real valued. It is not possible to get the conjugate symmetry of real numbers.

Result: The output image is not much clear as we can see in Fig. 7, and it is also not acceptable for the 
diagnostic purpose.
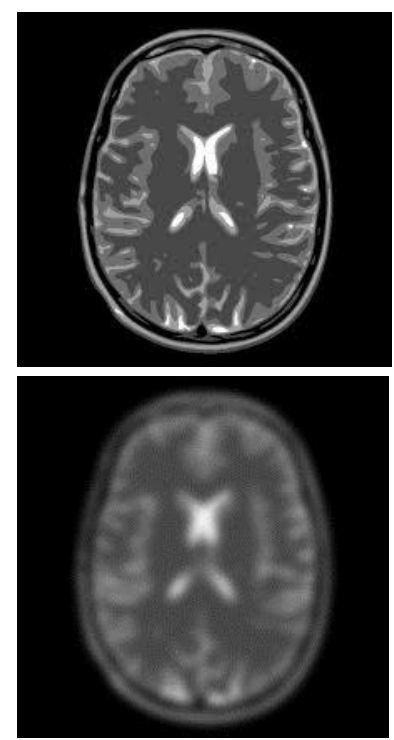

Fig.7. Original Phantom Image(U) and Reconstructed Phantom Image(D) after applying Phase Correction using Conjugate Symmetry

\section{Homodyne Reconstruction Algorithm}

Motivation: The main drawback in the previous approach (phase correction using conjugate symmetry algorithm) is that after doing the phase correction, the Fourier transform and inverse Fourier transform have to be computed multiple times to get the reconstructed image. This transform and inverse transform are considered as overhead for the reconstruction of an image. Homodyne algorithm overcomes this and requires the FT and IFT to be done only once.

Theory: This method uses an extra function called weighing function [72] that splits the real and imaginary part of the partial k-space data into anti symmetric and symmetric components. The main motive of this weighing function is to get the uniform distribution of spatial frequency in k-space data that are usually not uniform. The uniform distribution of spatial frequency in k-space data can be obtained by multiplying weighing function to the partial $\mathrm{k}$-space. The nature of weighing function can be seen in Fig. 8, you can refer [72] for weighing function. The algorithm takes input as partial kspace data and produces reconstructed image.

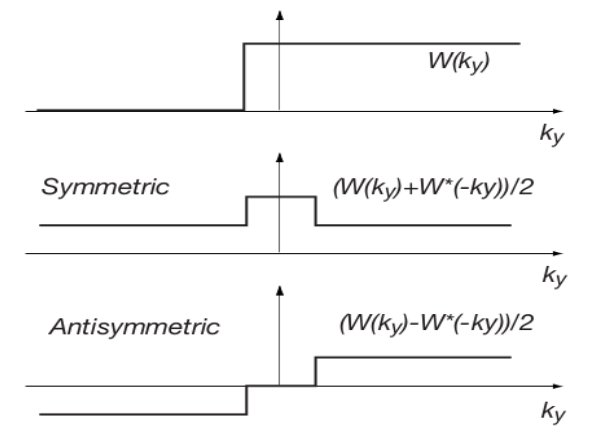

Fig.8. Weighing function is performing uniform distribution [72]

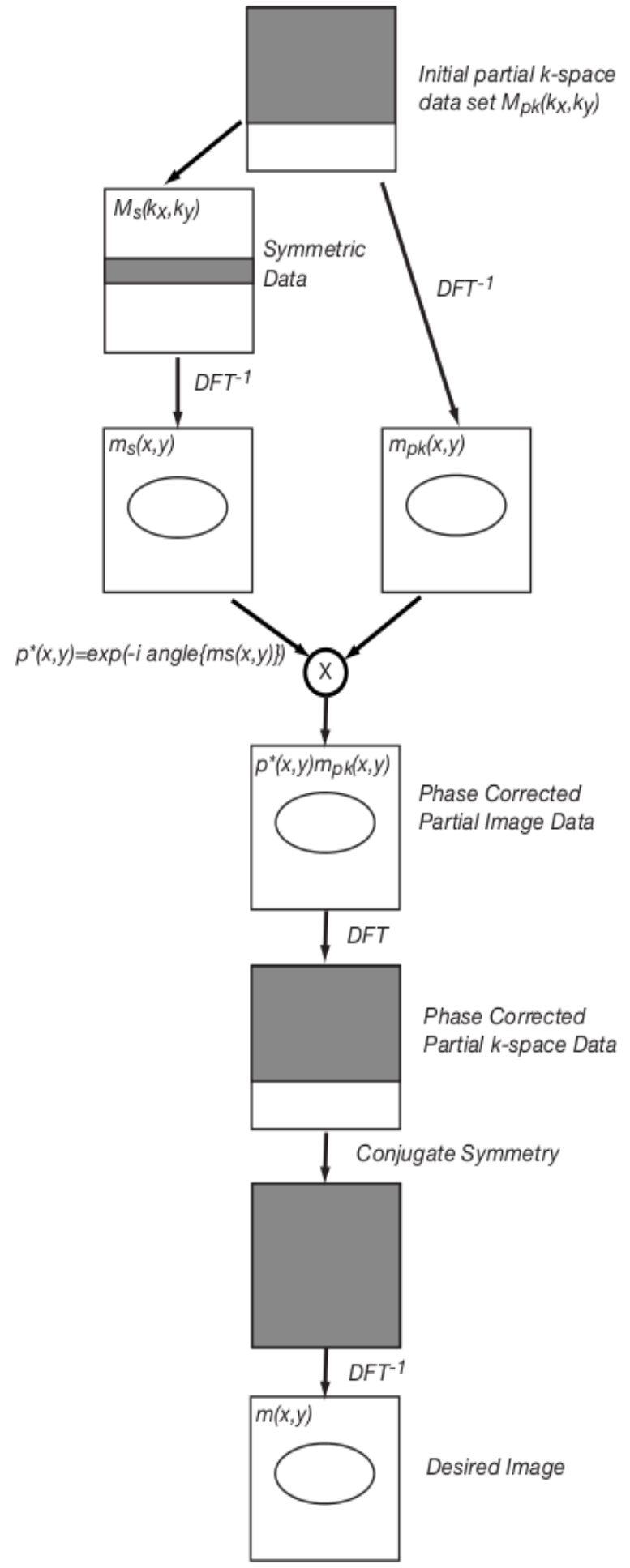

Fig.9. Pictorial Representation of Phase Correction using Conjugate Symmetry Algorithm [72]

Algorithm [75]:

It is similar to the "phase correction using conjugate symmetry" algorithm except for the weighing function factor. Fig. 10 illustrates the algorithm

Difference with Phase Correction using Conjugate Symmetry method: 
Both algorithms perform well when the variation of phase is minimal in both. The Homodyne algorithm performs phase correction after conjugate synthesis while phase corrections using conjugate symmetry perform the phase correction before conjugate synthesis.

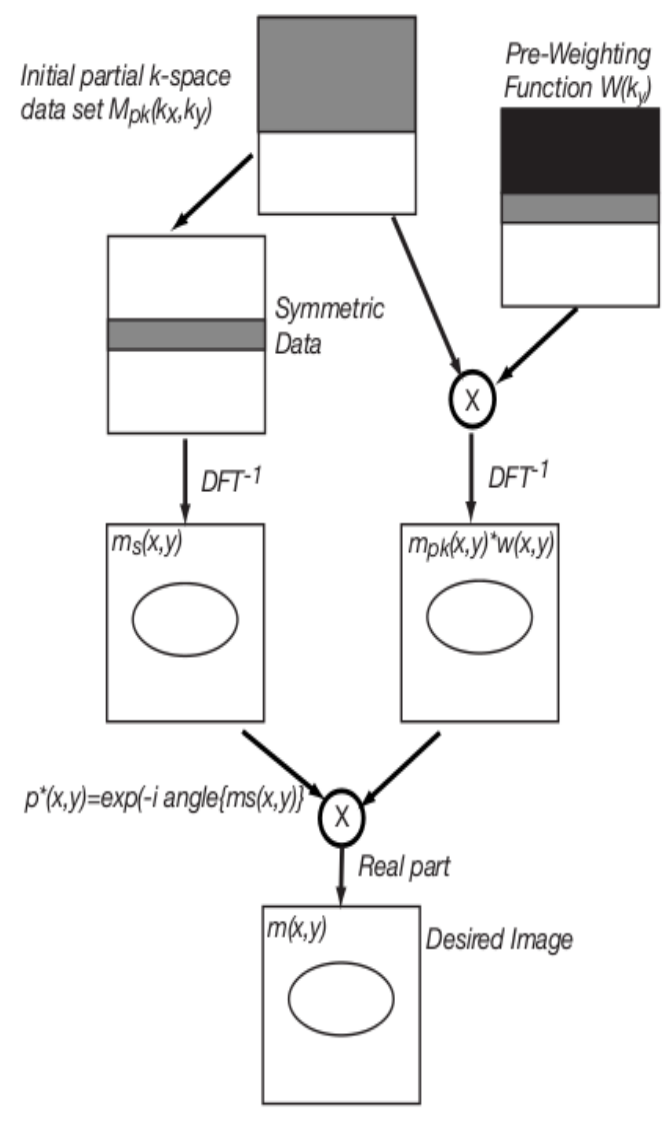

Fig.10: Pictorial Representation of Homodyne Reconstruction Algorithm [72]

Result: As we can see in Fig. 11 that output of Homodyne reconstruction algorithm is better than phase correction using conjugate symmetry method. The image is cleared and acceptable for the diagnostic purpose in 1990 's

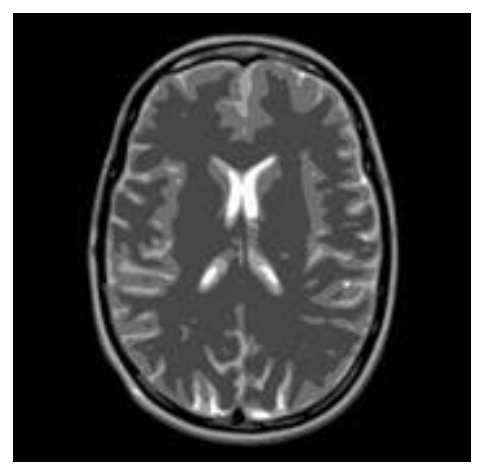

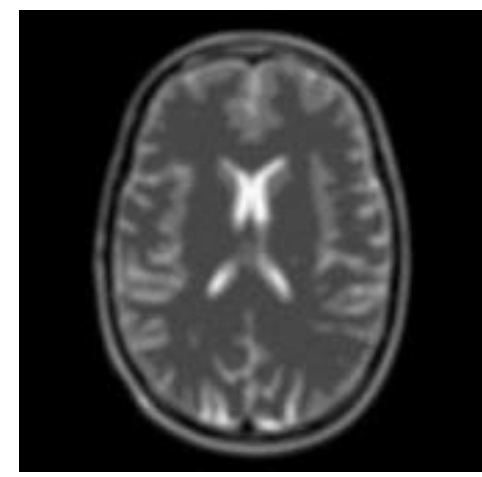

Fig.11. Original Phantom Image (U) and Reconstructed Phantom Image (D) after applying Homodye method

\section{Summary of Classical Reconstruction Algorithms}

Various reconstruction algorithms Zero Filling Reconstruction Method, Phase Correction using Conjugate Symmetry Method, Homodyne Reconstruction method, have been discussed. They all are Direct Methods (perform reconstruction in one pass only). They all work well when the phase varies slowly. They give unavoidable reconstruction errors when the k-space data contains almost all real values because conjugate symmetry is not possible for real values.

\section{RECENT RELATED WORKS IN MRI IMAGE RECONSTRUCTION}

Some remarkable work in the field of Image Reconstruction from MRI has been discussed in the previous sections $\mathrm{V}$ and section VI. Image reconstruction is still an active area of research. Time and again, scientists and engineers are contributing to this area. A few of the recent developments are reviewed briefly in the following:

\section{A. Alternating Minimization}

One of the recent works in image reconstruction is that by Kyle Herrity et al. [76]. They made an assumption that when the image is sparse we have only partial entry in PSF (Point Spread Function) [59]. They attempted to recover the magnetization distribution density that got lost due to permittivity and permeability towards the surrounding. Authors used an Iterative approach called "Alternating Minimization" for solving multiple linear equations efficiently. The minimization technique is used to bring down the number of missing coefficients. They discussed two models of Alternating Minimization algorithm such that one model follows the sparsity and other one follows Total Variation [77]. This algorithm is applied for MRI reconstruction which was then a comparatively latest imaging technology that is capable of resolving the image using spatial and temporal atomic scale. The authors concluded that their models provide better results than the other iterative techniques (e.g., ART) [78] when the image is sparse.

\section{B. Harmonic Analysis and Sparsity}


Alfred et al. [79] reviewed the theoretical results from sparse solutions of systems that are linear. Harmonic analysis of wavelets and applications of sparsity to signal and image processing are discussed. The sparsity based solution of linear equations in the application of image compression and image reconstruction are also discussed. Authors discussed various concepts like LU Decomposition, and Least Square Method of Applied Mathematics in signal processing. They also reviewed the results obtained from the linear equation of sparse image matrices and image reconstruction using projections (raw-data in CT scan).

\section{Signal Acquisition Protocol}

Candes et al. [80] presented how to reconstruct an image from numerical optimization from the full-length signal/image using the raw data ( $\mathrm{k}$-space) that is also called projections. They also described a very simple and efficient signal acquisition protocol that samples the signal which is independent of rate of change in projections. The final set obtained after the sampling allows improving the reconstruction power of sparse data, and permits better reconstruction of images.

\section{Stein's Unbiased Risk Estimator (SURE)}

Raich and Alfred [81] proposed sparse image reconstruction in radio astronomy. They proposed three sparse image reconstruction methods; first uses Stein's unbiased risk estimator(SURE) for selecting the hyperparameter for L1 estimator, and the other two, based on sparse prior, use empirical Bayes denoising. These three methods are simulated and found to produce better results than sparse Bayesian learning (SBL) [82]. They also explained the use of sparse priors in the Baye's framework empirically. Sparse prior value is much less than 1. Sparse prior is the magnetization intensity falling on the body. They examined three priors for the purpose of Baye's estimator [82] and compared to SBL, which itself is a Gaussian prior.

\section{E. Signal Modeling Approaches}

Jeffrey A. Fessler [83] discussed the elegance and flexibility of magnetic resonance imaging. Classically, the images from magnetic resonance imaging were reconstructed using 2Dimension and 3Dimension Fast Fourier Transformation. Those all reconstruction techniques were not efficient to give proper images because of the non uniform distribution of magnetic field, non Fourier effects and unknown Trajectories of k-space. The image reconstruction is not a single problem in magnetic resonance imaging but it is a combination of several problems that depends upon physical effect of MRI like hardware configuration.

They also presented a model which distributes the collected data (k-space) into small sub-set that is also called sub problems. The proposed models are Magnetic Resonance Signal Modeling, Measurement Modeling, and Linear Reconstruction Modeling. Magnetic resonance signal modeling gives good results as compared other primitive Inverse Fourier Transformation of the k-space data model.

\section{F. Non Iterative Algebraic Approach}

Jose and Govindan [84] proposed a new non-iterative algebraic approach for reconstructing images from projections. The idea they followed is to compute pixel values one by one from projections that passes through these pixels. The ray width and angle of projections are chosen in such a way that the first projection passes though only one pixel, and the second passes through one more unknown pixel, and the third passes through one more unknown pixel etc. With the help of triangular linear equations, the system of algebraic equations is formed. Triangular equation is solved in linear amount of time. This approach is also memory efficient because they are not storing the values iteratively.

\section{G. Sphere Shaped Support Vector Machine (SSSVM)}

Guo et al. [85] proposed SSSVM approach for classifying encephalic tissue in MRI reconstructed images. Most of the brain is made up of encephalic tissues which are highly irregular in shape. Classical method would not provide accurate reconstruction of brain image. SVM (Support Vector Machine) is used to reconstruct these complex images. It uses two form of SVM; first, SSSVM, which is applied for the 3D Image Reconstruction. Second, IFT (Image Fusion Technique) is applied for minimizing the loss of information in $\mathrm{k}$ space data. Authors concluded that SSSVM is a successful approach for the image reconstruction in which degree of irregularities is more.

\section{H. Temporal Resolution}

Temporal Resolution [86] refers to an imaging process where data collection takes place when the object is in motion; e.g., beating heart Image is captured using the phenomena of temporal resolution which involves multiple frames scanning at a time. Cinema Imaging is also one of the examples [87, 89] of temporal resolution.

It is assumed for Temporal Resolution that the $\mathrm{k}$-Space data is radically processed or sampled [88]. The sampling divides the temporal resolution in two ways on the basis of k-Space data; firstly, the k-space data collection can be improved (it is a hardware technique); secondly, the temporal resolution must utilize the high spatial frequency region.

Various concepts of Image Reconstruction that has been reviewed in this section are Alternating Minimization approach, SSSVM approach and many others Alternating Minimization is also used with fMRI for scanning that explained the functional nature of nerves, tissue, blood movement etc. Some of the most popular modern algorithms [72] for image reconstruction from k-space data like Projection onto Convex Set (POCS method), reconstruction using SVD, and Dictionary Learning method are discussed in detail in the next section (VII). The recent approaches that would discuss are iterative in nature that means a number of scans are required to rebuild data. 


\section{MODERn ALGORITHMS For IMAGE RECONSTRUCTION}

In the classical approaches, there is emphasis on phase variation but here only in POCS method significance to phase variation is given. Singular Value Decomposition (SVD) and Dictionary Learning are the modern approaches that rebuild the given k-space data efficiently. The modern approaches that we will discuss are all iterative in nature. There are number of iteration requires rebuilding data. These are complex than the classical algorithms that we discussed in section VI.

\section{A. Projection onto Convex Set Algorithm}

Input: Partial k-space data.

Output: Reconstructed Image.

Theory: It's a mathematical model which is used to determine point of convergence (or intersection) between two convex sets [90], where each set contains group of functions, here in our case first set contains determined phase constraint and other contain image constraint.

This approach guesses the k-space data by applying phase correction using conjugate symmetry algorithm (Section V, A) iteratively. As it is discussed, the most of the information about an image is near to central region. The central region is bounded for iteration with fix point in range $x_{1}$ to $x_{2}$ and $y_{1}$ to $y_{2}$ which can be seen in Fig. 12.

After several iterations, the correct $\mathrm{k}$-space data become nearly conjugate symmetric.

\section{Assumptions:}

$\mathrm{m}(\mathrm{x}, \mathrm{y})$ represents true image

$\mathrm{M}\left(\mathrm{k}_{\mathrm{x}}, \mathrm{k}_{\mathrm{y}}\right)$ represents full $\mathrm{k}$-space data

Since, $\mathrm{m}(\mathrm{x}, \mathrm{y})=\operatorname{IDFT}\left\{\mathrm{M}\left(\mathrm{k}_{\mathrm{x}}, \mathrm{k}_{\mathrm{y}}\right)\right\}$

$\mathrm{M}_{\mathrm{p}}\left(\mathrm{k}_{\mathrm{x}}, \mathrm{k}_{\mathrm{y}}\right)$ represents low frequency region, (central region)

A new sub k-space is defined from $\mathrm{k}_{\mathrm{x} 1}<=\mathrm{k}_{\mathrm{x}}<\mathrm{k}_{\mathrm{x} 2}$ can be given as:

$$
\begin{aligned}
& \mathrm{M}_{\mathrm{p}}\left(\mathrm{k}_{\mathrm{x}}, \mathrm{k}_{\mathrm{y}}\right)=\{ \\
& \mathrm{M}\left(\mathrm{k}_{\mathrm{x}}, \mathrm{k}_{\mathrm{y}}\right) ; \text { if }\left(\mathrm{k}_{\mathrm{x} 1}<=\mathrm{k}_{\mathrm{x}}<\mathrm{k}_{\mathrm{x} 2}\right) \\
& \text { 0; elsewhere }
\end{aligned}
$$$$
\text { \} }
$$

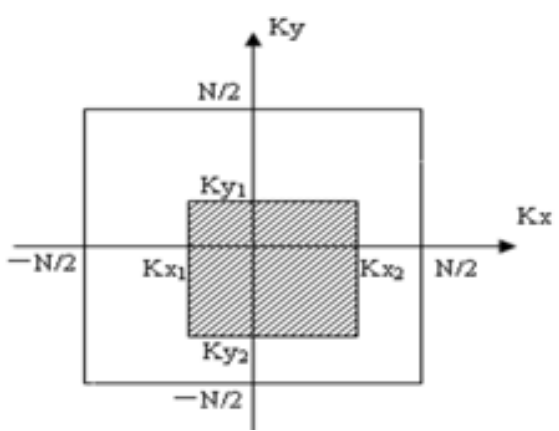

Fig.12. k-Space data emphasizing on Central Region [90]

\section{Algorithm [90]:}

1. Take input as k-space raw-data (matlab: ".m" file)

(a) If it is Conjugate Symmetric, goto step 4 (b) Else, go to step 2

2. Apply idft on $M_{p}$, we will get $m_{p}$, partial reconstructed image

$$
\mathrm{m}_{\mathrm{p}}(\mathrm{x}, \mathrm{y})=\operatorname{idft}\left(\mathrm{M}_{\mathrm{p}}\left(\mathrm{k}_{\mathrm{x}}, \mathrm{k}_{\mathrm{y}}\right)\right)
$$

3. Find phase constraint for each entry, after phase shift, the reconstructed intermediate image can be gien as

$$
\mathrm{m}_{\mathrm{r}}(\mathrm{x}, \mathrm{y})=\left|\mathrm{m}_{\mathrm{p}}(\mathrm{x}, \mathrm{y})\right| \exp (\mathrm{i} . \phi(\mathrm{x}, \mathrm{y}))
$$

And new k-space can be given as:

$$
\mathrm{M}_{\mathrm{r}}\left(\mathrm{k}_{\mathrm{x}}, \mathrm{k}_{\mathrm{y}}\right)=\operatorname{DFT}\left\{\mathrm{m}_{\mathrm{r}}(\mathrm{x}, \mathrm{y})\right\}
$$

Iterate step 2 and 3 until phase started varying slowly (The almost conjugate asymmetric k-space is obtained)

4. Do Inverse Fourier Transformation of new k-space, we'll get reconstructed image,

$$
\mathrm{m}_{\mathrm{r}}=\operatorname{IDFT}\left\{\mathrm{M}_{\mathrm{r}}\left(\mathrm{k}_{\mathrm{x}}, \mathrm{k}_{\mathrm{y}}\right)\right\}
$$

here, $\mathrm{m}_{\mathrm{r}}$ is the reconstructed image.

Fig. 14 illustrates the POCS method.

Pros: It produces better quality images than that of direct methods because it changes the conjugate symmetry and phase correction iteratively which process the image and spatial frequency domain again and again and then produces contrast image.

Cons: If the phase varies rapidly even after several iterations. It doesn't produce good result. POCS method also fails in filling entries in k-space if the k-space is quite more asymmetrical.

Result: As we can see in Fig. 13 that output of POCS Algorithm which is much better than other non-iterative techniques.
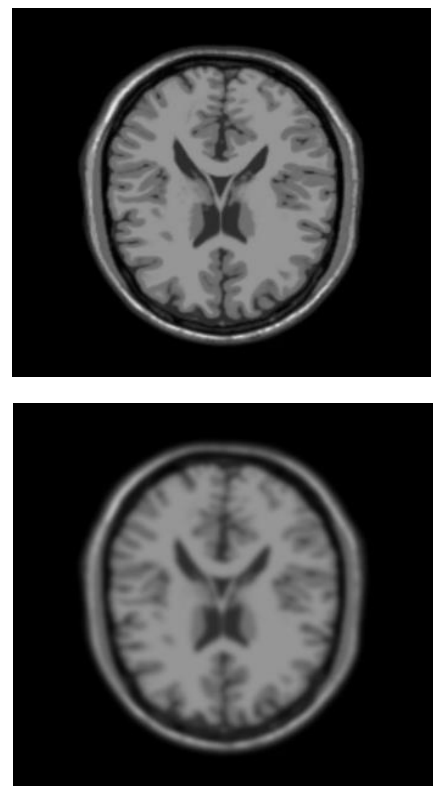

Fig.13. Original Brain Phantom Image (U) and Reconstructed Phantom Image (D) after applying POCS Reconstruction Algorithm 


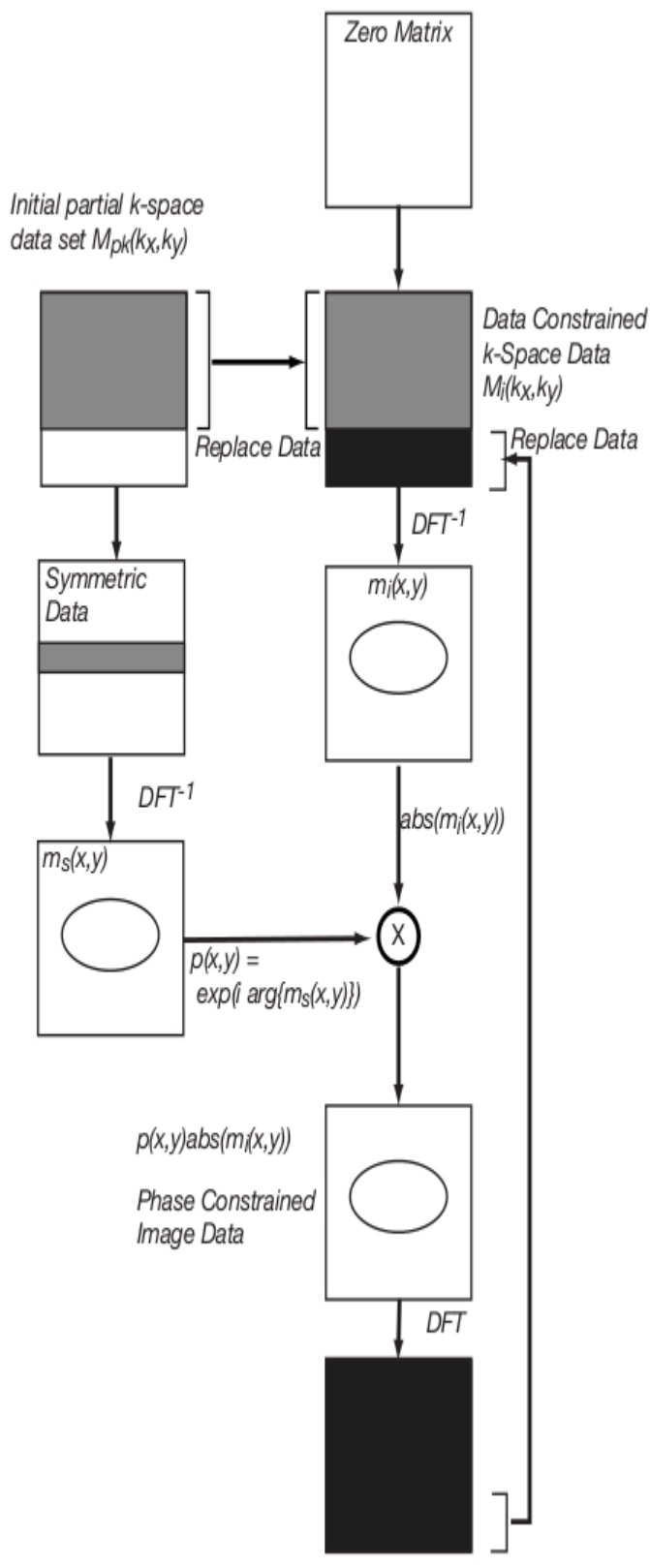

Fig.14. Pictorial Representation of POCS Algorithm [72]

\section{B. Reconstruction using Singular Value Decomposition}

SVD (Single Value Decomposition) [91] is factorization of complex/real matrix which has very useful implementations in Signal Processing/ Image Processing.

\section{Fundamentals of Singular Value Decomposition:}

$\mathrm{M}=\mathrm{U} \boldsymbol{\Sigma} \mathrm{V}^{*}, \mathrm{M}$ is Given Matrix

$\mathrm{U}$ is $\mathrm{m} \mathrm{x} \mathrm{m}$ size unitary matrix,

$\boldsymbol{\Sigma}$ is an $\mathrm{m} \mathrm{x} \mathrm{n} \mathrm{diagonal} \mathrm{matrix,}$

$\mathrm{V}^{*}$ is conjugate transpose of $\mathrm{V}$ unitary matrix of size $\mathrm{n}$ $\mathrm{x} \mathrm{n}$

Its application is of finding pseudo inverse of a matrix. $\mathrm{M}^{+}=\mathrm{V} \Sigma^{+} \mathrm{U}^{*}$
$\Sigma^{+}$is obtained by replacing every non-zero diagonal entry by its reciprocal and transposing the resulting matrix.

\section{Reconstruction Algorithm using SVD}

The algorithm takes input as partial $\mathrm{k}$-space data and produces reconstructed image.

\section{Algorithm [91]:}

As it is know, k-space is a matrix of complex number of phase encoded signal in Fourier Space. The k-space in the frequency domain or spatial can be written as:

$S\left(k_{x}, k_{y}\right)=\frac{1}{M N} \sum_{x=0}^{M-1} \sum_{y=0}^{N-1} X(x, y) e^{-i 2 \pi\left(\frac{k_{x} x}{M}+\frac{k_{y} y}{N}\right)} e^{i \phi(x, y)}$

In the terms of SVD k-space can be written as:

$$
S_{M N \times 1}=A_{M N \times M^{2}} \times X_{M^{2} \times 1}
$$

A is the matrix of Fourier coefficient whose values are phase corrected, $\mathrm{S}$ is the $\mathrm{k}$-space data, and $\mathrm{X}$ is the value of intensity that need to be reconstructed.

The inference of $\mathrm{X}$ is used to evaluate the pseudo inverse of the matrix A. The idea of SVD in the reconstruction is to make the matrix regular. Regular signifies that there should be only one value corresponding to each row and column.

A pre-determined Fourier Coefficient matrix (See Fig. 14) exists through practical purposes and do pseudo inverse on a matrix after phase error.

Fig. 16 illustrates the detail about SVD method.

Pros: It gives quite better result and if there is priory knowledge about the Fourier coefficient matrix, it gives best result. If we have highly configured system then it's a best among all that we discussed above.

Cons: It is a complex process, its time and space complexity is very high. Suppose we are having four variables SVD image size variables $\mathrm{k}$ and $\mathrm{L}$. Let $\mathrm{k}=128$, $\mathrm{L}=84$, size is $128^{2} * 84^{2} * 2 * 8=1.7 \mathrm{~Gb}$ memory, which is quite more even for Modern Computer Systems.

Result: It gives good result and images reconstructed from this algorithm are acceptable. Its result into better quality econstructed images (Fig. 15) that of images reconstructed by POCS method.

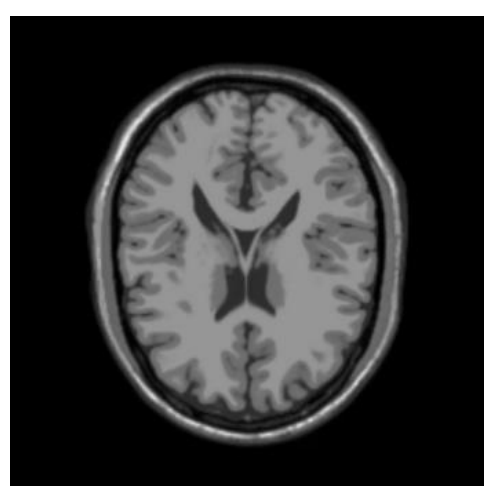




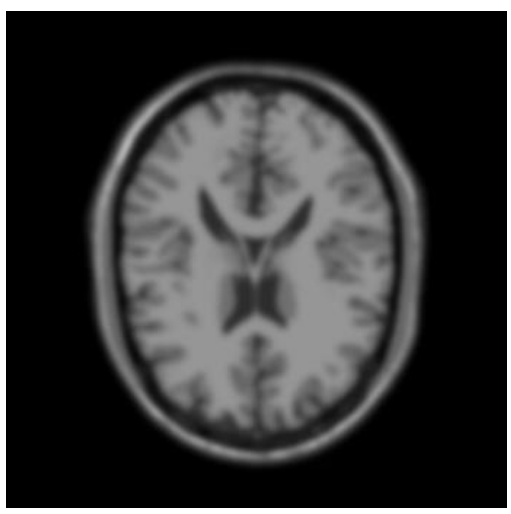

Fig.15. Original Brain Phantom Image (U) and Reconstructed Phantom Image (D) after applying SVD method

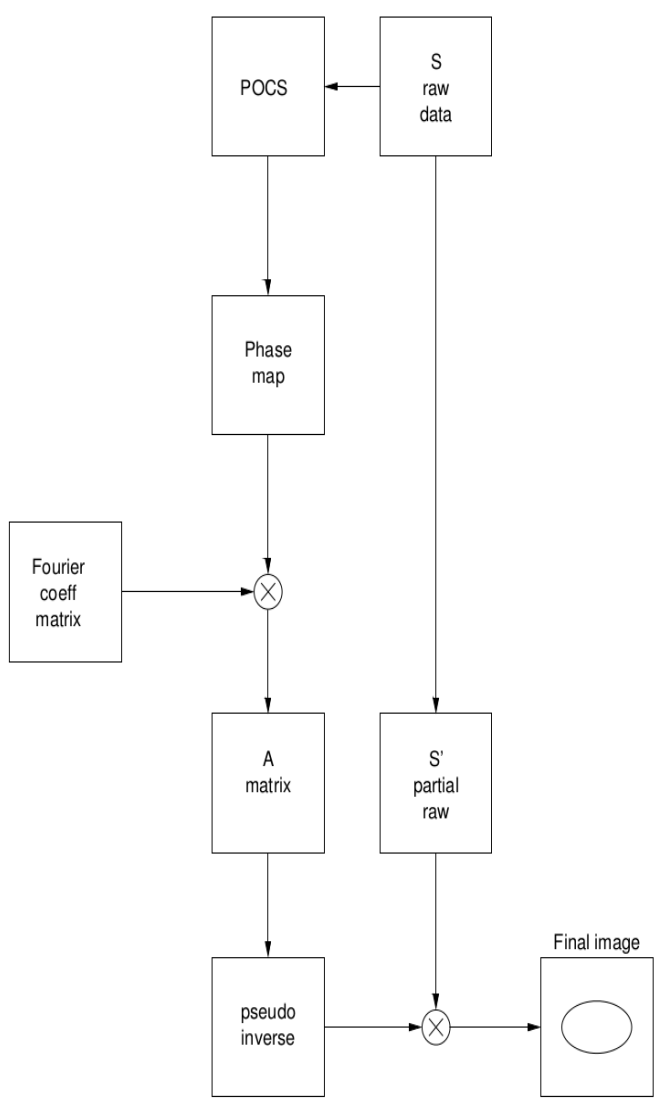

Fig.16. Pictorial Representation of SVD image reconstruction method [91]

\section{Reconstruction using Dictionary Learning}

It is used for sparse representations of images/signals. It assembles the collection of atom. These atoms are collection of column vectors of some fixed length. Let the length of atom be $\mathrm{N}$ here and the total number of atoms are $\mathrm{K}$. A dictionary $\mathrm{D}$ can be created of size $\mathrm{N} x \mathrm{~K}$.

$\mathrm{X}$ is a vector and represents a signal/image in terms of linear combination of some of the Dictionary atoms and Sparse Random Vector can be given as

$$
\mathrm{X}=\mathrm{D} . \mathrm{w} \text {; }
$$

Where 'w' is a Sparse Random Vector [92]
Theory: The quality of reconstructed images can be improved in two ways, Firstly, increase the number of atoms in dictionary learning [93] upto blocks. Secondly, divide the dictionary learning scheme in two levels, a low resolution learning and high resolution learning. Low resolution is applicable to the concept of sparse coding while high resolution is applicable for updating the Image. This is a new method proposed in 2011 and can be further improved.
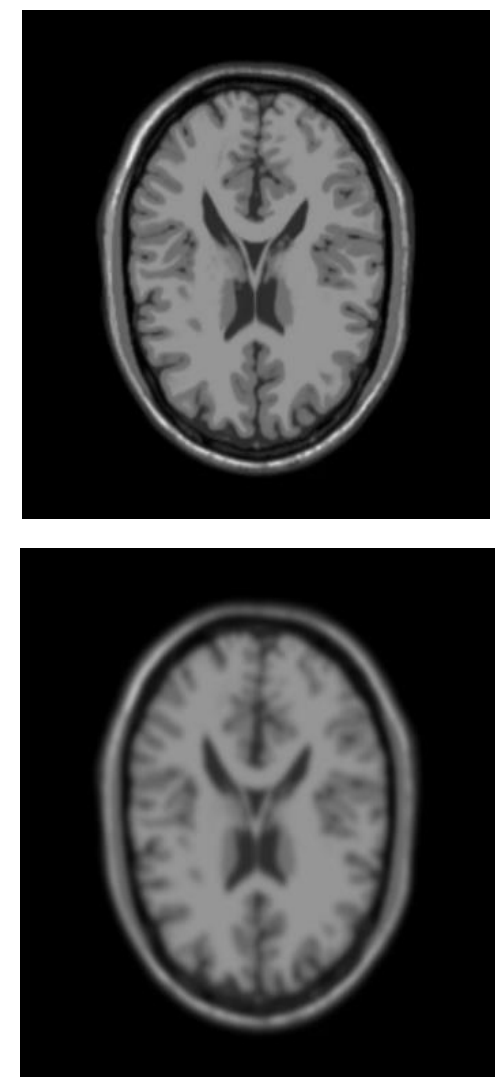

Fig.17. Original Brain Phantom Image (U) and Reconstructed Phantom Image (D) after applying SVD method

Results: The dictionary learning algorithm achieves better quality images (Fig. 17) as compared to the POCS method. It reconstructs images which are quite similar to SVD method. The best thing is with Dictionary Learning method it is efficient than SVD method.

\section{Summary of Modern Reconstruction Algorithms}

We have discussed POCS Reconstruction Method, SVD method, Dictionary learning method. Dictionary learning is the modern approach; we can use already build dictionary that can be used for reconstructing images. Dictionary Learning gives better result when the input data is sparsed. SVD method involves lot of mathematics for reconstructing images. Dictionary learning is also one of the wide area for research. There's a good scope in Image Reconstruction field. 
In this survey paper, the basics of MRI have been briefly reviewed. It is a multi-disciplinary area involving physics, mathematics, computational sciences etc. All these algorithms for reconstruction come under the computational sciences. The input raw data for these algorithms is $\mathrm{k}$-space data. Various modern magnetic resonance imaging techniques like fMRI, diffusion MRI were also discussed. The concept of phase correction, phase error and types of k-space data has been introduced. The concept of Expectation Maximization, Sensitive Encoding, Level Set Method and many others have been reviewed in the classical works in image reconstruction. The section on Classical research works mainly focused on the enhancement of gradient coding and reduction in the scan time. The concept of Alternating Minimization, Signal Modeling Approaches, and, SSSVM have been reviewed in the category of recent works in image reconstruction.

The algorithm, zero filled method is a trivial method, it works fine when full k-space is available, but with partial $\mathrm{k}$-space it gives unwanted blurring. The phase correction using conjugate symmetry, homodyne algorithm and projection onto convex set (POCS) work well only if variation in phase in the image is low. When the degree of phase variation is high, these algorithms give ghosting effect in an image. The Singular Value Decomposition (SVD) works better even if phase variation is there in $\mathrm{k}$ space data. Dictionary Learning Image Reconstruction is one of the recent techniques for reconstructing image; it reduces the time of scanning. If the degree of variation of phase in k-space data is high, then images cannot be reconstructed properly even with POCS method. It is also observed that the images can be reconstructed properly by including the concept of total variation [94] in the POCS method.

\section{ACKNOWLEDGMENT}

I would like to express my special thanks of gratitude and deep regards to Mr. Chandan Kumar Jhamb and Miss. Jyotsna Chanda, Mtech, NIT Surathkal, India; for their exemplary support, and constant encouragement throughout the survey. I would also like to thank $\mathrm{Mr}$. Mahipal Singh Parmar, Mr. Amar Maroti Kaldate and Mr. Sanjeet Sharma Mtech, NIT Calicut, India; for their valuable and constructive suggestions during the survey.

\section{REFERENCES}

[1] Hongmei Zhu "Medical Image Processing Overview" University of Calgary, Toronto, Ontario M5T 3J1.

[2] MS Nizam BJJ Abdullah Kwan-Hoong Ng, Azlan C Ahmad "Magnetic resonance imaging: Health effects and safety", Proceedings of the International Conference on NonIonizing Radiation at UNITEN ICNIR2003, (ICNIR2003), 20th October 2003.

[3] Purcell EM, Torrey HC, and Pound RV "Resonance absorption by nuclear magnetic moments in a solid" Phys Rev 69: 37-38, 1946., MIT, Massachusetts, December 24, 1945.

[4] Ming Zhao, Daniel A. Beauregard, Louiza Loizou, Bazbek Davletov \& Kevin M. Brindle "Non-invasive detection of apoptosis using magnetic resonance imaging and a targeted contrast agent", MRC Laboratory of Molecular Biology, Hills Road, Cambridge, UK,Nature Medicine 7, 1241 - 1244 (2001), doi:10.1038/nm1101124.

[5] Abi Berger, science editor "Article on Magnetic Resonance Imaging" BMJ. Jan 5, 2002; 324(7328): 35. PMCID: PMC1121941, US National Library of Medicine National Institutes of Health.

[6] Kay Powell "The Difference Between MRI \& CT (CAT Scans)", Article on Medical and Dental Expenses: Topic 502, From the IRS, Holland.

[7] Robert Holgersson and Mikael Svärd "Reconstruction of Echo Planar Images used in Functional MRI", Institute of Technology, Linkopin University, Master's Thesis at the Department of Biomedical Engineering, LiU-IMT-EX359, February 2004.

[8] Joseph P. Hornak, Book Title "The Basics Of MRI", chapter-6, Director of the Magnetic Resonance Laboratory, Rochester Institute of Technology, Rochester, NY 146235604.

[9] H. Bruder, H. Fischer, H.-E. Reinfelder and F. Schmitt"Image reconstruction for echo planar imaging with nonequidistant k-space sampling", Article first published online: 18 NOV 2005 DOI: 10.1002/mrm.1910230211.

[10] G.T. Herman "Image Reconstruction From Projections", Medical Image Processing Group, Department of Radiology, University of Pennsylvania, Philadelphia, PA 19104, USA gabor@mipg.upenn.edu Available online 2 May 2002, Volume 1, Issue 1, April 1995, Pages 3-18, doi:10.1006/rtim.1995.1002.

[11] D. R. Smith, Willie J. Padilla, D. C. Vier, S. C. NematNasser, and S. Schultz "Composite Medium with Simultaneously Negative Permeability and Permittivity", Phys. Rev. Lett. 84, 4184 - Published 1 May 2000, Department of Physics, University of California, San Diego, 9500 Gilman Drive, La Jolla, California 920930319.

[12] Jeffrey G. Jarvik, MD, MPH; and Richard A. Deyo, MD, $\mathrm{MPH}$ "Diagnostic Evaluation of Low Back Pain with Emphasis on Imaging", University of Washington, Seattle, Washington, Ann Intern Med. 2002; 137(7):586-597. doi:10.7326/0003-4819-137-7-200210010-00010.

[13] Stephan G. Nekolla, Axel Martinez-Moeller, Antti Saraste "PET and MRI in cardiac imaging: from validation studies to integrated applications" Published online: 23 December 2008 Springer-Verlag 2008 Eur J Nucl Med Mol Imaging (2009) 36 (Suppl 1):S121-S130 DOI 10.1007/s00259008-0980-1.

[14] Kevin Cleary, Charles Nguyen "State of the art in surgical robotics: Clinical applications and technology challenges", Volume 6, Issue 6, pages 312-328, 2001, Article first published online: 4 APR 2002 DOI: 10.1002/igs.10019, Copyright $\left({ }^{\circ} 2002\right.$ Wiley-Liss.

[15] Uduma Felix U, Fokam Pius G "Magnetic Resonance Imaging (Mri), The Prefered Evaluation Tool In Soft Tissue Sarcoma: Literature Review, Demonstrated With A Case Report", Journal of Asian Scientific Research, 2012, vol. 2, issue 2, pages 87-99.

[16] K. Tadakuma, L.M. DeVita, J.S. Plante, Y. Shaoze and S. Dubowsky, IEEE Fellow "The experimental study of a precision parallel manipulator with binary actuation: With application to MRI cancer treatment" 2008. ICRA 2008. ..., 2008 - ieeexplore.ieee.org.

[17] Helene Benvenistea, Steve Blackbandc "MR microscopy and high resolution small animal MRI: applications in neuroscience research", Medical Department, Brookhaven 
National Laboratory, NY 11793, USA, Received 13 February 2002, Accepted 7 June 2002, Available online 10 September 2002, doi:10.1016/S0301-0082(02)00020-5.

[18] Mark A. Horsfield1, Derek K. Jones"Applications of diffusion-weighted and diffusion tensor MRI to white matter diseases - a review" Article first published online: 5 DEC 2002 DOI: 10.1002/nbm.787 Copyright (C) 2002 John Wiley \& Sons, Ltd.

[19] David Berreby "Scanning Brains for Insights on Racial Perception" Article publish at NewYork Times, Science Section, September 5, 2000, Copyright (C) 2000 The New York Times Co. Reprinted with permission.

[20] Douglas C. Noll "Notes on Physics of Magnetic Resonance Imaging" Co-Director, Functional MRI Laboratory Department of Biomedical Engineering, University of Michigan, United States of America, Fall2006, Notes-1.

[21] D Moratal, Vallés-Luch, MSc, L Martí-Bonmatí, ME Brummer "k-Space tutorial: an MRI educational tool for a better understanding of k-space", Biomedical Imaging and Intervention Journal Tutorial, Received 8 January 2008; received in revised form 21 February 2008; accepted 10 March 2008.

[22] A.J. Bohris, U. Goerke, P.J. McDonald, M. Mulheron†, B. Newling, B. Le Page "A broad line NMR and MRI study of water and water transport in portland cement pastes", published on ELSEVIER Available online 29 January 1999, doi:10.1016/S0730-725X(98)00072-1.

[23] Moriel NessAiver "Magnetic Resonance Imaging", Article at Simply Physics, Science Journal of Doctrates, NessAiver, Ph.D., 3406 Labyrinth RdBaltimore, MD 21215.

[24] Seon Yeong Lee and Dympna Gallagher "Assessment methods in human body composition", Clinical nutrition and metabolic care 2008; 11(5):566-572. doi:10.1097/MCO.0b013e32830b5f23.

[25] Turner, RE, Snider, RF, Fleming, DG "SPIN RELAXATION OF HYDROGEN-ATOM ISOTOPES VIA ELECTRON-SPIN EXCHANGE WITH PARAMAGNETIC GASES", Journal Physical Review A, Volume 41 Pagination 1505-1516 Date Published Feb 1990 ISBN Number 1050-2947.

[26] John Scampini, "Introduction to Magnetic Resonance Imaging (MRI)", TUTORIAL 4681, Executive Director in the Industrial Communications and Ultrasound Business Unit @ May 10, 2010, Maxim Integrated Products, Inc.

[27] B.J. Casey, Matthew Davidson and Bruce Rosen "Functional magnetic resonance imaging: Basic principles of and application to developmental science", Blackwell Publishers Ltd. 200 Developmental Science 5:3 (2002), pp 301-309.

[28] Thulborn KR, Waterton JC, Matthews PM, Radda GK "Oxygenation dependence of the transverse relaxation time of water protons in whole blood at high field". Biochim. Biophys. Acta 714 (2): 265-70. doi:10.1016/0304-4165(82)90333-6. PMID 6275909.

[29] John C. Gore "Principles and practice of functional MRI of the human brain", J Clin Invest. Jul 1, 2003; 112(1): 4 9. doi: 10.1172/JCI200319010.

[30] SUSUMU MORI AND PETER B. BARKER "Diffusion Magnetic Resonance Imaging:Its Principle and Applications", Department of Radiology Anat Rec (New Anat) 257:102-109, 1999. r 1999 Wiley-Liss, Inc.

[31] Aaron G. Filler "The History, Development and Impact of Computed Imaging in Neurological Diagnosis and Neurosurgery: CT, MRI, and DTI", Received 12 July 2009 06:43 UTC; Posted 13 July 2009 Nature Precedings. doi:10.1038/npre.2009.3267.5.

[32] S. J. Holdsworth, S. Skare, and R. Bammer "On the application of phase correction and use of k-space entropy in partial Fourier diffusion-weighted EPI" Lucas MRS/I Center, Stanford University, Stanford, CA, United States.

[33] Martin Uecker, Shuo Zhang, Dirk Voit, Alexander Karaus, Klaus-Dietmar Merboldt and Jens Frahm "Real-time MRI at a resolution of $20 \mathrm{~ms}$ ", NMR in Biomedicine, Volume 23, Issue 8, pages 986-994, October 2010Article first published online: 27 AUG 2010 OI: 10.1002/nbm.1585.

[34] A Haase, J Frahm, D Matthaei, W Hänicke, KD Merboldt "FLASH imaging: rapid NMR imaging using low flip angle pulses",Max-Planck-Institut für biophysikalische Chemie, Germany, Volume 67, Issue 2, Journal of Magnetic Resonance 67 (2): 258-266. doi:10.1016/00222364(86)90433-6.

[35] Schnall MD, Bolinger L, Renshaw PF, Haselgrove JC, Subramanian VH, Eleff SM, Barlow C, Leigh JS Jr, Chance B."Multinuclear MR imaging: a technique for combined anatomic and physiologic studies", published at ncbi, Radiology. 1987 Mar; 162(3):863-6.

[36] Ann Scott "Interventional and Intraoperative Magnetic Resonance Imaging", Alberta Heritage Foundation for Medical Research,Edmonton, AB T5J 4A7 CANADA, March 2004 IP 17: Information Paper

[37] E. Mark Haacke "Susceptibility Weighted Imaging" Opening new doors to clinical applications of magnetic resonance imaging 5th Edition (2006) Department of Radiology, Wayne State University 3990 John R Detroit, MI 48202.

[38] Mansur G. Abdullah,Michael C. Anderson "Magnetic susceptibility" Article Written by: The Editors of Encyclopædia Britannica, www.britannica.com.

[39] Gujar, Sachin K; Maheshwari, Björkman-Burtscher, Isabella MD, Sundgren, Pia C MD, "Magnetic Resonance Spectroscopy", Journal of Neuro-Ophthalmology, United State of AmericaSeptember 2005 - Volume 25 - Issue 3 pp 217-226.

[40] RP Wagner, HK Mitchell "Genetics and metabolism" [BOOK] - 1955 - Am Genetic Assoc, Lucid survey of biochemical basis of inheritance.

[41] Ian Boomer, "Isotopes: theory, principles and practicalities", Stable Isotope \& Luminescence Laboratory, Senior Research Fellow (SILLA) University of Birmingham, Edgbaston BirminghamB15 2TT UK.

[42] Paul Suetens (ed): "Fundamentals of Medical Imaging (2nd edition)" [BOOK] European Journal of Nuclear Medicine and Molecular Imaging, Dec 2010.

[43] Douglas C. Noll and Bradley P. Sutton "Gridding Procedures for Non-Cartesian K-space Trajectories" Dept. of Biomedical Engineering, University of Michigan, Ann Arbor, MI, USA.

[44] Denis Hoa "K-space exploration", article at http://www.imaios.com.

[45] Dr Jeremy Jones, Dr Usman Bashir "Article on k-space" on Radiopaedia.org, Royal Marsden Hospital, London, United Kingdom, http://radiopaedia.org/articles/k-space.

[46] Rahul Tayal "MRI Reconstruction" EN 250: Medical Imaging, Spring 2009, Laboratory for Engineering ManMachine Systems.

[47] James Meakin "Introduction to MRI Acquisition", FMRIB Physics Group, FSL Course, Bristol, September 2012.

[48] Sarah K. Patch "k-Space Data Preprocessing for Artifact Reduction in MR Imaging", Multidimensional Image Processing, Analysis, and Display: RSNA Categorical Course in Diagnostic Radiology Physics 2005; pp 73-87.

[49] Deans, Stanley R. (1983), "The Radon Transform and 
Some of Its Applications", New York: John Wiley \& Sons.

[50] Anthony J. Devaney "Generalized Projection-Slice Theorem for Fan Beam Diffraction Tomography", doi: $10.1177 / 016173468500700306$ Ultrasonic Imaging July 1985 vol. 7 no. $3264-275$.

[51] Dr. Matthew D. Robson, John C. Gore and R. Todd Constable "Measurement of the point spread function in MRI using constant time imaging", Article first published online: 12 DEC 2005 DOI: 10.1002/mrm.1910380509 Magnetic Resonance in Medicine Volume 38, Issue 5, pages 733-740, November 1997.

[52] Jiang Hsieh "Computed Tomography: Principles, Design, Artifacts, and Recent Advances" Published: 2009, DOI:10.1117/3.817303, eISBN: 9780819480422 | Print ISBN13: 9780819475336.

[53] Lauterbur PC (1973). "Image Formation by Induced Local Interactions: Examples of Employing Nuclear Magnetic Resonance". Nature 242 (5394): 190-1. Bibcode: 1973Natur.242.190L. doi: 10.1038/242190a0.

[54] Yuan Cheng, C.Forbes Dewey "Discrete tomography by bayesian labeling with its efficient algorithm" C.Forbes Dewey Proc. SPIE 4121, Mathematical Modeling, Estimation, and Imaging, doi:10.1117/12.402448, October 2000.

[55] Klaas P. Pruessmann, Markus Weiger, Markus B. Scheidegger, and Peter Boesiger"SENSE: Sensitivity Encoding for Fast MRI", Wiley-Liss, Inc publish in Magnetic Resonance in Medicine 42:952-962 (1999).

[56] Gylson Thomas and V K Govindan, A sequency domain filtered-backprojection algorithm for fast computation of tomographic images, \#rd Int nat. Conf on Computational Intelligence, robotics and autonomous systems, National University of Singapore, Dec 2005.

[57] Megha Borse, S.B. Patil, B.S.Patil "LITERATURE SURVEY FOR 3D RECONSTRUCTION OF BRAIN MRI IMAGES", International Journal of Research in Engineering and Technology, Volume: 02 Issue: $11 \mid$ Nov2013.

[58] Andrei V Bronnikov "A filtering approach to image reconstruction in 3D SPECT", published in Physics in Medicine and Biology, Volume 45, Number 92000 Phys. Med. Biol. 452639 doi:10.1088/0031-9155/45/9/315.

[59] Che-Yen Wen,Chien-Hsiung Lee "Point spread functions and their applications to forensic image restoration Introduction" ,Forensic Science Journal 2002;1:15-26, Department of Forensic Science, Central Police University, Taiwan ROC.

[60] http://photo.net/learn/optics/mtf/.

[61] Alejandro Ribés and Francis Schmitt "Linear Inverse Problems in Imaging" IEEE SIGNAL PROCESSING MAGAZINE JULY 2008 Digital Object Identifier 10.1109/MSP.2008.923099, IEEE Xplore. Downloaded on October 20, 2008 at 15:41.

[62] Magdeleine Dinguirard and Philip N. Slater "Calibration of Space-Multispectral Imaging Sensors: A Review", Optical Sciences Center, University of Arizona, Tucson, AZUSA Received 7 July 1998, Revised 24 November 1998, Available online 21 June 1999 Volume 68, Issue 3, June 1999, Pages 194-205.

[63] Gylson Thomas and V. K. Govindan, Computationally efficient filtered-back projection algorithm for topographic image reconstruction using Walsh transform, Journal of Visual communication and image representation, Vol 17, Issue 3, June 2006.

[64] Jong Chul Ye, Yoram Bresler, Pierre Moulin "A SelfReferencing Level-Set Method for Image Reconstruction from Sparse Fourier Samples" Punblished in International
Journal of Computer Vision December 2002, Volume 50, Issue 3, pp 253-270.

[65] Richard Ttsai and Sstanley Oosher "level set methods and their applications in image science", University of California, Berkley, Cambridge University Press.

[66] Laura B. Montefusco, Damiana Lazzaro, "Nonlinear Filtering for Compressed Sensing Sparse Image Reconstruction" Department of Mathematics University of Bologna, Italy Serena Papi CIRI-ICT University of Bologna, Italy.

[67] Michael Lustig, David L. Donoho Member, Juan M. Santos Member and John M. Pauly "Compressed Sensing MRI", Packard 210, Published in Department of Electrical Engineering, 350 Serra Mall, Stanford University Stanford CA, 94305.

[68] Nick Kingsbury, Julian Magarey "Wavelet Transforms in Image Processing", Signal Analysis and Prediction Applied and Numerical Harmonic Analysis 1998, pp $27-$ 46, Springer Copyright 1998 DOI 10.1007/978-1-46121768-8_2.

[69] Martin S. Banks, Benjamin R. Stephens, E.Eugenie Hartmann "The development of basic mechanisms of pattern vision: Spatial frequency channels $\imath^{\zeta}$ ", Journal of Experimental Child Psychology Volume 40, Issue 3, December 1985, Pages 501-527.

[70] M. W. K. Kan, L. H. T. Leung, W. Wong, and N. Lam, "Radiation dose from cone beam computed tomography for image-guided radiation therapy," International Journal of Radiation Oncology Biology Physics, vol. 70, no. 1, pp 272-279, 2008.

[71] W. De Vos, J. Casselman, G.R.J. Swennen1, "Cone-beam computerized tomography (CBCT) imaging of the oral and maxillofacial region: A systematic review of the literature" Published at ELSEVIER, Accepted 27 February 2009, Volume 38, Pages 609-625.

[72] Karla Miller "Partial k-space Reconstruction", Lecture Notes, FMRIB Centre, University of Oxford, Nuffield Department of Clinical Neurosciences, University of Oxford, UK.

[73] Bin Rong Wu, Satoshi Ito, Yoshitsugu Kamimura and Yoshifumi Yamada "Zero-filling Technique in Fresnel Transform Image Reconstruction for MR Image Denoising", Progress In Electromagnetics Research Symposium 2005, Hangzhou, China, August 22-26.

[74] Christopher K. Anand, Paul M. Margosian, Francis H. Bearden"Two-dimensional phase-conjugate symmetry reconstruction for 3d spin-warp, echo-planar and echovolume magnetic resonance imaging", Philips Medical Systems (Cleveland), Inc., Publication type: Grant, Publication date 25 Jun 2002.

[75] Douglas C. Noll, Dwight G. Nishimura, Member, IEEE, and Albert Macovski, Fellow, IEEE "Homodyne Detection in Magnetic Resonance Imaging", IEEE TRANSACTIONS ON MEDICAL IMAGING, VOL. IO, NO. 2, JUNE 1991.

[76] Kyle Herrity, Raviv Raich and AlfredO.HeroIII. "Blind reconstruction of sparse images with unknown point spread function" In HeroIII Department of EECS, University of Michigan, Ann Arbor, MI 48109-2122 School of EECS, Oregon State University, Corvallis, OR 97331-5501, pages 128-137, May 2008.

[77] Ludwig Ritschl1, Frank Bergner1, Christof Fleischmann2 and Marc Kachelrieß1 "Improved total variation-based CT image reconstruction applied to clinical data", Phys. Med. Biol. 56 1545. doi:10.1088/0031-9155/56/6/003, Received 24 August 2010, in final form 12 January 2011. Published 16 February 2011. Institute of Physics and Engineering in 
Medicine.

[78] Herman, Gabor T., "Fundamentals of computerized tomography: Image reconstruction from projection", published by Academic Press, 1980, Advances in Computer Vision and Pattern Recognition, 2nd edition, Springer, 2009.

[79] Alfred M., David L., Donoho Bruckstein and Michael Elad. "From sparse solutions of systems of equations to sparse modeling of signals and images related databases" Publisher: Society for Industrial and Applied Mathematics, DOI: 10.1137/060657704(3):471-81, July 2007.

[80] Candes Emmanuel J. and B. Wakin Michael. "An introduction to compressive sampling" IEEE Signal Processing Magazine, March 2008, Pages-10, ISBN 10535888/08/\$25.002008IEE.

[81] Raviv Raich, Alfred Michael Ting "Sparse image reconstruction using sparse priors" Electrical Engineering and Computer Science, University of Michigan, Ann Arbor, MI 48109-2122, USA.

[82] D. P. Wipf and B. D. Rao, "Sparse Bayesian learning for basis selection," IEEE Trans. Signal Processing, vol. 52, no. 8, pp. 2153-2164, 2004.

[83] Jeffrey A. Fessler "MODEL-BASED IMAGE RECONSTRUCTION FOR MRI" Systems Lab of the ECE Division within the Electrical Engineering and Computer Science, GE Healthcare, University of Michigan, 4431 EECS Bldg., 1301 Beal Ave.

[84] Johnes Jose and Govindan V.K.; Image Reconstruction from Projections using Triangular Linear System of Equations; International Journal of Engineering Research \& Technology (IJERT); Vol. 3 Issue 1, January - 2014; ISSN: 2278-0181.

[85] Guo L1, Wang L, Wu Y, Yan W, Shen X. "Research on 3D modeling for head MRI image based on immune sphere-shaped support vector machine.", Conf Proc IEEE Eng Med Biol Soc. 2007;2007:1082-5, Engineering in Medicine and Biology Society, 2007. EMBS 2007. 29th Annual International Conference of the IEEE

[86] Patrik Brynolfsson "Using Radial k-space Sampling and Temporal Filters in MRI to Improve Temporal Resolution", Medical Physics Conference, Department of Radiation Sciences Umea University, Umea, Sweden 2010.

[87] Dr Yuranga Weerakkody and Dr Usman Bashir "Temporal resolution" Article at Radiopedia, MBBS MMed FRCR Department of Radiology, Royal Marsden Hospital, London, United Kingdom.

[88] http://mri-q.com/rf-transmit-coils.html.

[89] http://mri-info.net/welcome/?page_id=634.

[90] Jiaming Chen, Lu Zhang, Yuemin Zhu, Jianhua Luo "MRI Reconstruction From 2D Partial k-Space Using POCS Algorithm",College of Life Science \& Technology, Shanghai Jiao Tong University, 200240, Shanghai, P.R.China, 978-1-4244-2902-8/09/\$25.00 @2009 IEEE.

[91] Fadi Yaacoub 1,2, Antoine Abche 1, Elie Karam 1 and Yskandar Hamam "MRI Reconstruction Using SVD in the Least Square Sense", 21st IEEE International Symposium on Computer-Based Medical Systems, 1063-

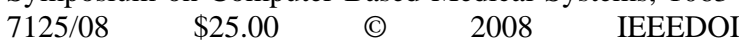
10.1109/CBMS.2008.662.3.

[92] Ron Rubinstein, Alfred M. Bruckstein and Michael Elad "Dictionaries for Sparse Representation Modeling" Senior Member, IEEE, IEEE PROCEEDINGS, VOL. 5 Israel Institute of Technology, Haifa 32000.

[93] Ying Song, Zhen Zhu, Yang Lu1, Qiegen Liu1 and Jun Zhao1, "Reconstruction of magnetic resonance imaging by three-dimensional dual-dictionary learning", Published at Wiley, Volume 71, Issue 3, pages 1285-1298, March 2014.

[94] Guoqiang Yu, Liang Li, Jianwei Gu, and Li Zhang "Total Variation Based Iterative Image Reconstruction", Department of Application Science, Nuctech Company Limited, Beijing, P.R. China 100084 CV B I A 2005, LNCS 3765, pp. 526-534, 2005.c Springer-Verlag Berlin Heidelberg 2005.

\section{Authors' Profiles}

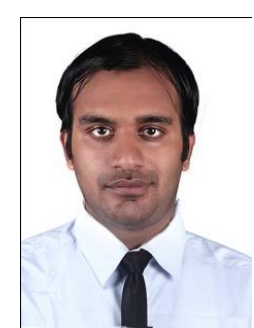

Mr. Tanuj Kumar Jhamb, Master Degree Scholar, Department of Computer Science and Engineering, National Institute of technology Calicut, Kerala, India. $\mathrm{He}$ is recruited as Edison Engineer in General Electric, India. He received his Bachelor Degree from Punjabi University, Patiala, Punjab, India in 2013.

Weblink: nitc.academia.edu/tanujjhamb

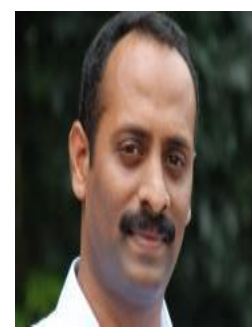

Mr. Vinith Rejathalal, Faculty, Department of Computer Science and Engineering, National Institute of technology Calicut, Kerala India. $\mathrm{He}$ is also a $\mathrm{PhD}$ research scholar in the Institute. He received his Master Degree in Computer Science from Amrita Vishwa Vidyapeetham, Coimbatore, India. His areas of interest are Pattern Recognition, Machine Learning, and Neuroimaging.

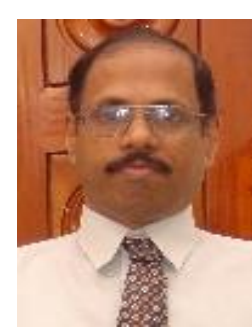

Dr. V.K. Govindan received Bachelors and Masters degrees in electrical engineering from National Institute of technology (NIT) Calicut in the year 1975 and 1978, respectively. He received $\mathrm{PhD}$ from IISc Bangalore, India.. He has more than 35 years of teaching experience at UG and PG levels and he was the Professor and Head of the Department of Computer Science and Engineering, NIT Calicut during the years 2000 to 2005 . He was Dean Academic of NIT Calicut during 2011 to 2014. He is currently working as Professor in the Department of Computer Science and Engineering at NIT Calicut, India. His research areas include Image processing, pattern recognition, machine learning, data compression, document imaging and operating systems. He has 155 research publications including 80 international Journal papers, and authored 20 books. He has produced seven $\mathrm{PhDs}$, adjudicated several $\mathrm{PhDs}$ and refereed many papers for Journals and conferences. 